\title{
ON $\mathrm{K}_{3}$ OF WITT VECTORS OF LENGTH TWO OVER FINITE FIELDS
}

\author{
THOMAS GEISSER
}

\begin{abstract}
We prove that for $W_{2}\left(\mathbb{F}_{q}\right)$ the Witt vectors of length two over the finite field $\mathbb{F}_{q}$, we have $\mathrm{K}_{3}\left(W_{2}\left(\mathbb{F}_{p^{f}}\right)\right)=\left(\mathbb{Z} / p^{2}\right)^{f} \oplus \mathbb{Z} /\left(p^{2 f}-1\right)$ in characteristic at least 5 and $\mathrm{K}_{3}\left(W_{2}\left(\mathbb{F}_{3 f}\right)\right)=(\mathbb{Z} / 9)^{f-1} \oplus(\mathbb{Z} / 3)^{2} \oplus \mathbb{Z} /\left(3^{2 f}-1\right)$ for $(3, f)=1$. The result is proved by using the identity $\mathrm{K}_{3}\left(W_{2}\left(\mathbb{F}_{q}\right)\right)=H_{3}\left(S L\left(W_{2}\left(\mathbb{F}_{q}\right)\right)\right)$ and calculating the right term with a group homology spectral sequence. Some information on the spectral sequence is achieved by using the action of the outer automorphism of $S L$ on the homology groups and recent results on Kgroups of local rings and the ring of dual numbers over finite fields.
\end{abstract}

\section{INTRODUCTION}

Some of the higher algebraic K-groups which can be explicitly calculated are the groups $\mathrm{K}_{i}\left(\mathcal{O}_{\mathfrak{p}} / \mathfrak{p}^{n}\right)$ for $\mathcal{O}_{\mathfrak{p}}$ the ring of integers in a local field. The prime-to- $p$ part is given by the prime-to- $p$ part of $K_{i}\left(\mathcal{O}_{\mathfrak{p}} / \mathfrak{p}\right)$ by Suslin [21]. The groups $K_{2}\left(\mathcal{O}_{\mathfrak{p}} / \mathfrak{p}^{n}\right)$ have been calculated by Dennis and Stein [5]. In the totally ramified case, the groups $\mathrm{K}_{i}\left(\mathbb{F}_{q}[t] / t^{2}\right)$ have been determined by Hesselholt and Madsen [10] and in the unramified case, Evens and Friedlander [7] proved $\mathrm{K}_{3}\left(\mathbb{Z} / p^{2}\right)_{p}=\mathbb{Z} / p^{2}$ for $p \geq 5$. In this paper we extend this result in two ways. The main theorem is:

Theorem 1.1. a) Let $p \geq 5$ then

$$
\mathrm{K}_{3}\left(W_{2}\left(\mathbb{F}_{p^{f}}\right)\right)=\left(\mathbb{Z} / p^{2}\right)^{f} \oplus \mathbb{Z} /\left(p^{2 f}-1\right) .
$$

b) Let $(3, f)=1$ then

$$
\mathrm{K}_{3}\left(W_{2}\left(\mathbb{F}_{3^{f}}\right)\right)=(\mathbb{Z} / 9)^{f-1} \oplus(\mathbb{Z} / 3)^{2} \oplus \mathbb{Z} /\left(3^{2 f}-1\right) .
$$

Part b) includes $K_{3}(\mathbb{Z} / 9)_{3}=(\mathbb{Z} / 3)^{2}$, conflicting the result of [1]. The characteristic 3 case is of particular interest, because it is known that $\pi_{3}(\mathrm{im} J)_{3}$, the homotopy group of the image of the $J$ homomorphism, gives a direct summand $\mathbb{Z} / 3$ of $K_{3}(\mathbb{Z})=\mathbb{Z} / 48$ and of $K_{3}\left(\mathbb{Z}_{3}, \mathbb{Z}_{3}\right)=\mathbb{Z}_{3} \oplus \mathbb{Z} / 3$. On the other hand one knows by Panin [15] that $K_{3}\left(\mathbb{Z}_{3}, \mathbb{Z} / 3\right)=\lim _{\leftarrow} K_{3}\left(\mathbb{Z} / 3^{n}, \mathbb{Z} / 3\right)$. So the question arises at which level the image of $J$ occurs for the first time in the inverse system. The above theorem says that it arises at the earliest possible level $n=2$.

The proof of the theorem uses the identity

$$
\mathrm{K}_{3}\left(W_{2}\left(\mathbb{F}_{q}\right)\right)=H_{3}\left(S L\left(W_{2}\left(\mathbb{F}_{q}\right)\right)\right) .
$$

The right hand term is then calculated as in [7], [1], [14] and [19], using the Hochschild Serre spectral sequence associated to the extension

$$
0 \rightarrow V \rightarrow S L\left(W_{2}\left(\mathbb{F}_{q}\right)\right) \rightarrow S L\left(\mathbb{F}_{q}\right) \rightarrow 0
$$

Key words and phrases. Higher algebraic K-theory, Hochschild Serre spectral sequence, Charlap-Vasquez theory, group cohomology, linear groups, local rings.

* supported by Deutsche Forschungsgemeinschaft. 
Some $E^{2}$-terms in this spectral sequence have been calculated by Lluis-Puebla [14] and Friedlander and Parshall [8]. We need the following additional results.

On the one hand, a main lemma 4.2 tells us that the map $\mathrm{K}_{3}\left(\mathcal{O}_{\mathfrak{p}}, \mathbb{Z}_{p}\right) \rightarrow$ $\mathrm{K}_{3}\left(\mathcal{O}_{\mathfrak{p}} / \mathfrak{p}^{2}\right)$ is surjective. This gives an upper bound on the number of generators of $\mathrm{K}_{3}\left(\mathcal{O}_{\mathfrak{p}} / \mathfrak{p}^{2}\right)$, because the groups $\mathrm{K}_{3}\left(\mathcal{O}_{\mathfrak{p}}, \mathbb{Z}_{p}\right)$ have been calculated by Levine [13] and Bökstedt and Madsen [2].

On the other hand, we use the action of the outer automorphism of $S L$ on the terms of the spectral sequence to show that some differentials vanish. Using the calculation of $\mathrm{K}_{i}\left(\mathbb{F}_{q}[t] / t^{2}\right)$ of Hesselholt and Madsen [10], which admits a spectral sequence with the same $E_{2}$-terms, this suffices to calculate $K_{3}\left(\mathcal{O}_{\mathfrak{p}} / \mathfrak{p}^{2}\right)$ in characteristic at least 5 .

In characteristic 3 we have to calculate an explicit differential in the spectral sequence. This takes the second half of the paper and follows ideas of Evens and Friedlander[7].

Notation: $\mathbb{F}_{q}$ denotes the field with $q=p^{f}$ elements, $W_{n}(R)$ the Witt vectors of length $n$ over $R$ and $W(R)$ all Witt vectors. For a group $V, V^{*}$ denotes the dual group $\operatorname{Hom}(V, \mathbb{Q} / \mathbb{Z})$ and $V_{p}$ the $p$-part of $V . V_{n}\left(\mathbb{F}_{q}\right)$ are the $n \times n$-matrices of trace zero over $\mathbb{F}_{q}$, and $V\left(\mathbb{F}_{q}\right)$ is the direct limit of the $V_{n}\left(\mathbb{F}_{q}\right)$. We will sometimes write $V$ if the field in question is clear from the context. For an $R$-module $V$ over the ring $R, \Lambda_{R}^{n} V$ is the $n$-th exterior power and $S_{R}^{n} V$ the $n$-th symmetric power.

I would like to thank the following people for many helpful conversations and their patience in listening to me: B.Gross, L.Hesselholt, M.Levine, V.Snaith. I would also like to thank C.Stahlke for his help with the computer calculations and the Harvard Department of Mathematics for its hospitality during my stay.

\section{K-GROUPS AND GROUP COHOMOLOGY}

For any ring $R$ and $n \geq 1$ the K-groups and K-groups with coefficients are defined to be

$$
\begin{aligned}
\mathrm{K}_{n}(R) & =\pi_{n}\left(B G L^{+}(R)\right), \\
\mathrm{K}_{n}(R, \mathbb{Z} / m) & =\pi_{n}\left(B G L^{+}(R), \mathbb{Z} / m\right),
\end{aligned}
$$

where $G L(R)=\lim _{\rightarrow} G L_{n}(R), B$ is the classifying space and + Quillen's plusconstruction. As $B \vec{S} L(R)^{+}$is the universal covering of $B G L^{+}(R)$, we get for $n \geq 2$ : $\mathrm{K}_{n}(R)=\pi_{n}\left(B S L^{+}(R)\right)$. We also have short exact sequences

$$
0 \rightarrow \mathrm{K}_{n}(R) / m \rightarrow \mathrm{K}_{n}(R, \mathbb{Z} / m) \rightarrow{ }_{m} \mathrm{~K}_{n-1}(R) \rightarrow 0
$$

If $\mathrm{K}_{2}(R)_{p}=0$, the Hurewitz theorem shows

$$
\mathrm{K}_{3}(R)_{p}=H_{3}(S L(R))_{p}, \quad \mathrm{~K}_{3}(R, \mathbb{Z} / p)=H_{3}(S L(R), \mathbb{Z} / p)
$$

and the latter determines the number of generators of the former. Thus we will be interested in the low dimensional homology groups of $S L(R)$.

Note that by duality we have

$$
H_{n}(S L(R), \mathbb{Z} / p)=H^{n}(S L(R), \mathbb{Z} / p)^{*} .
$$

If $G$ is torsion, we get from the long exact sequence to the short exact sequence $0 \rightarrow \mathbb{Z} \rightarrow \mathbb{Q} \rightarrow \mathbb{Q} / \mathbb{Z} \rightarrow 0$ and duality that $H^{1}(G)=0$ and that for $n \geq 2$

$$
H^{n}(G)=H^{n-1}(G, \mathbb{Q} / \mathbb{Z})=H_{n-1}(G)^{*}
$$


If $R$ is finite, the groups $S L_{n}(R)$ are finite and thus $S L(R)$ is torsion, so we can also use cohomology groups to calculate $K$-groups.

For $V$ an abelian group, we have [3, V 6.6]

$$
H_{*}(V, \mathbb{Z} / p)=\Lambda_{\mathbb{Z} / p}(V / p V) \otimes \Gamma_{\mathbb{Z} / p}\left({ }_{p} V\right) .
$$

If $V$ is $p$-torsion, we have $H_{1}(V)=V, H_{2}(V)=\Lambda^{2} V$ [3, V 6.4]. From the long cohomology sequence associated to the sequence $0 \rightarrow \mathbb{Z} \rightarrow \mathbb{Z} \rightarrow \mathbb{Z} / p \rightarrow 0$ we get

$$
V^{*}=H^{1}(V, \mathbb{Z} / p) \stackrel{\delta}{\sim} H^{2}(V),
$$

and the two dual sequences

$$
0 \rightarrow H^{2}(V)=\delta V^{*} \rightarrow H^{2}(V, \mathbb{Z} / p) \stackrel{\delta}{\rightarrow} H^{3}(V)=\Lambda^{2} V^{*} \rightarrow 0
$$

and

$$
0 \rightarrow H_{2}(V)=\Lambda^{2} V \stackrel{\pi}{\longrightarrow} H_{2}(V, \mathbb{Z} / p) \stackrel{\partial}{\rightarrow} H_{1}(V)=V \rightarrow 0 .
$$

In terms of the bar resolution the map $\pi$ is given by $\pi(u \wedge v)=[u \mid v]-[v \mid u]$ for $u \wedge v \in \Lambda^{2} V=H_{2}(V)$ and $\partial$ is given by $\partial[u \mid v]=\frac{[u]-[u+v]+[v]}{p}$. The map $\pi$ is split by $[u \mid v] \mapsto \frac{u \wedge v}{2}$ and $\partial$ is split by $\rho:[v] \mapsto \sum_{j=0}^{p-1}[v \mid j v]$.

Finally we get

$$
H_{3}(V)=\Lambda^{3} V \oplus S^{2} V=H^{4}(V)^{*}
$$

As we are interested in Witt vectors of length two $W_{2}\left(\mathbb{F}_{q}\right)$ over finite fields, we will consider the low terms of the spectral sequences associated to the short exact sequence induced by reduction modulo $p$ :

$$
0 \rightarrow K \rightarrow S L\left(W_{2}\left(\mathbb{F}_{q}\right)\right) \rightarrow S L\left(\mathbb{F}_{q}\right) \rightarrow 0
$$

If one identifies $K$ with the trace zero matrices over $\mathbb{F}_{q}$, then the action of $S L\left(\mathbb{F}_{q}\right)$ is the adjoint action.

The sequence gives rise to the Hochschild-Serre spectral sequences

$$
\begin{aligned}
E_{p, q}^{2}(\mathbb{Z})=H_{p}\left(S L\left(\mathbb{F}_{q}\right), H_{q}\left(V\left(\mathbb{F}_{q}\right)\right)\right) & \Rightarrow H_{p+q}\left(S L\left(W_{2}\left(\mathbb{F}_{q}\right)\right)\right) \\
E_{p, q}^{2}(\mathbb{Z} / p)=H_{p}\left(S L\left(\mathbb{F}_{q}\right), H_{q}\left(V\left(\mathbb{F}_{q}\right), \mathbb{Z} / p\right)\right) & \Rightarrow H_{p+q}\left(S L\left(W_{2}\left(\mathbb{F}_{q}\right)\right), \mathbb{Z} / p\right)
\end{aligned}
$$

and similarly for cohomology.

Lemma 2.1. Let $M$ be the group of all matrices over $\mathbb{F}_{q}, V$ be the trace zero matrices and $i>0$. Then we have

$$
H_{i}\left(S L\left(\mathbb{F}_{q}\right), V\right)=H_{i}\left(G L\left(\mathbb{F}_{q}\right), V\right)=H_{i}\left(G L\left(\mathbb{F}_{q}\right), M\right) .
$$

Proof: If $(n, q-1)=1$, the map det $: G L_{n}\left(\mathbb{F}_{q}\right) \rightarrow \mathbb{F}_{q}^{*}$ is $\operatorname{split}$ by $x \rightarrow \operatorname{diag}(x, x, \ldots, x)$ and the action of $\mathbb{F}_{q}^{*}$ on $H_{*}\left(S L_{n}\left(\mathbb{F}_{q}\right), V_{n}\right)$ induced by conjugation is trivial. As $\mathbb{F}_{q}^{*}$ has order prime to $p$ and $V_{n}$ is $p$-torsion, the spectral sequence

$$
H_{i}\left(\mathbb{F}_{q}^{*}, H_{j}\left(S L_{n}\left(\mathbb{F}_{q}\right), V_{n}\right) \Rightarrow H_{i+j}\left(G L_{n}\left(\mathbb{F}_{q}\right), V_{n}\right)\right.
$$

shows that $H_{*}\left(G L_{n}\left(\mathbb{F}_{q}\right), V_{n}\right)=H_{*}\left(S L_{n}\left(\mathbb{F}_{q}\right), V_{n}\right)$ and this carries over to the limit, proving the first equation.

If $(n, p)=1$, then the map tr $: M \rightarrow \mathbb{F}_{q}$ is split as a $G L\left(\mathbb{F}_{q}\right)$-map by $x \mapsto$ $\operatorname{diag}\left(\frac{x}{n}, \ldots \frac{x}{n}\right)$, and we have

$$
H_{i}\left(G L_{n}\left(\mathbb{F}_{q}\right), M_{n}\right)=H_{i}\left(G L_{n}\left(\mathbb{F}_{q}\right), V_{n}\right) \oplus H_{i}\left(G L_{n}\left(\mathbb{F}_{q}\right), \mathbb{F}_{q}\right)
$$


According to Quillen's result [16], the right hand side vanishes in the limit for $i>0$, proving the second equation.

The following terms of the above spectral sequence are known:

Proposition 2.2.

$$
\begin{aligned}
& \text { a) } \quad H_{i}\left(S L\left(\mathbb{F}_{q}\right), \mathbb{Z}\right)_{p}=0 \quad i>0 \\
& \text { b) } \quad H_{i}\left(S L\left(\mathbb{F}_{q}\right), V\right)=(\mathbb{Z} / p)^{f} \quad i \geq 2 \text { even } \\
& \text { c) } H_{i}\left(S L\left(\mathbb{F}_{q}\right), \Lambda^{2} V\right)=\begin{array}{ll}
0 & \text { otherwis } \\
0 & i=0,1
\end{array} \\
& \text { d) } H_{0}\left(S L\left(\mathbb{F}_{q}\right), S^{2} V\right)=(\mathbb{Z} / p)^{f} \\
& \text { e) } H_{0}\left(S L\left(\mathbb{F}_{q}\right), \Lambda^{3} V\right)=(\mathbb{Z} / p)^{f}
\end{aligned}
$$

Proof: a) [16, theorem 6]

b) By lemma 2.1 and duality we have

$$
H_{i}\left(S L\left(\mathbb{F}_{q}\right), V\right)=H_{i}\left(G L\left(\mathbb{F}_{q}\right), M\right)=H^{i}\left(G L\left(\mathbb{F}_{q}\right), M^{*}\right)^{*} .
$$

As $M=M^{*}$, the result follows from [8, prop. 1.6]

c) $[14$, theorems $2.3 \mathrm{e}, 2.4 \mathrm{~b}]$ or $[12$, théorème 3.4$]$

d), e) [14, theorem $2.4 \mathrm{c}]$

Remark: As [14] only contains sketches of proofs, we like to mention that the results of this paper remain valid if in d) and e) we only know that the homology groups have $p$-rank at least $f$.

But the compositions

$$
S^{2} V \stackrel{a b}{\longrightarrow} M \stackrel{t r}{\longrightarrow} \mathbb{F}_{q} \quad \text { and } \quad \Lambda^{3} V \stackrel{a b c-b a c}{\longrightarrow} M \stackrel{t r}{\longrightarrow} \mathbb{F}_{q}
$$

are $S L\left(\mathbb{F}_{q}\right)$-invariant. Composing them with linear forms $\chi: \mathbb{F}_{q} \rightarrow \mathbb{F}_{p}$, we see that $H^{0}\left(S L\left(\mathbb{F}_{q}\right), S^{2} V^{*}\right)$ and $H^{0}\left(S L\left(\mathbb{F}_{q}\right), \Lambda^{3} V^{*}\right)$ have dimension at least $f$ over $\mathbb{Z} / p$.

If we denote $H_{2}\left(S L\left(\mathbb{F}_{q}\right), \Lambda^{2} V\right)$ by $H$ we thus get

Corollary 2.3. a) The low terms in the spectral sequence $H_{p}\left(S L\left(\mathbb{F}_{q}\right), H_{q}(V)\right)_{p} \Rightarrow$ $H_{p+q}\left(S L\left(W_{2}\left(\mathbb{F}_{q}\right)\right)\right)_{p}$ are

\begin{tabular}{l|c|c|c|c|c}
3 & $(\mathbb{Z} / p)^{2 f}$ & & & & \\
\hline 2 & 0 & 0 & $H$ & & \\
\hline 1 & 0 & 0 & $(\mathbb{Z} / p)^{f}$ & 0 & $(\mathbb{Z} / p)^{f}$ \\
\hline 0 & $\mathbb{Z}$ & 0 & 0 & 0 & 0 \\
\hline & 0 & 1 & 2 & 3 & 4
\end{tabular}

b) The low terms in the spectral sequence $H_{p}\left(S L\left(\mathbb{F}_{q}\right), H_{q}(V, \mathbb{Z} / p)\right) \Rightarrow H_{p+q}\left(S L\left(W_{2}\left(\mathbb{F}_{q}\right)\right), \mathbb{Z} / p\right)$ are

\begin{tabular}{l|c|c|c|c|c}
3 & $(\mathbb{Z} / p)^{2 f}$ & & & & \\
\hline 2 & 0 & 0 & $(\mathbb{Z} / p)^{f} \oplus H$ & & \\
\hline 1 & 0 & 0 & $(\mathbb{Z} / p)^{f}$ & 0 & $(\mathbb{Z} / p)^{f}$ \\
\hline 0 & $\mathbb{Z} / p$ & 0 & 0 & 0 & 0 \\
\hline & 0 & 1 & 2 & 3 & 4
\end{tabular}




\section{K-GROUPS OF LOCAL RINGS}

In this section we recall some results on K-groups of dual numbers and local rings and relate them to the Lichtenbaum-Quillen conjectures.

By Suslin [21] we know that for a complete local ring $\mathcal{O}_{\mathfrak{p}}$ with quotient field $\mathbb{F}_{q}$ and $m$ prime to $p$ we have

$$
\mathrm{K}_{i}\left(\mathcal{O}_{\mathfrak{p}}, \mathbb{Z} / m\right)=\mathrm{K}_{i}\left(\mathbb{F}_{q}, \mathbb{Z} / m\right)
$$

Thus we will be only interested in the $p$-part of K-groups, as the prime to $p$-part is known by Quillen [16, theorem 8].

Similarly, Panin [15] has shown that for a complete discrete valuation ring of mixed characteristic

$$
\mathrm{K}_{i}\left(\mathcal{O}_{\mathfrak{p}}, \mathbb{Z} / p^{n}\right)=\lim _{\leftarrow} \mathrm{K}_{i}\left(\mathcal{O}_{\mathfrak{p}} / \mathfrak{p}^{r}, \mathbb{Z} / p^{n}\right)
$$

which allows us to relate K-groups of local rings to K-groups of their quotients.

The following two theorems have been proved by comparison of K-theory with topological cyclic homology:

Theorem 3.1. [10] Let $k$ be a finite field of characteristic $p \neq 2$, then

$$
\begin{aligned}
\mathrm{K}_{2 n}\left(k[t] /\left(t^{2}\right)\right)_{p} & =0 \\
\mathrm{~K}_{2 n-1}\left(k[t] /\left(t^{2}\right)\right)_{p} & =\bigoplus_{(i, 2)=1} W_{s_{i}}(k) .
\end{aligned}
$$

Here $s_{i}$ is given by $i p^{s_{i}-1} \leq n<i p^{s_{i}}$.

Theorem 3.2. [2] Let $\mathcal{O}_{\mathfrak{p}}$ be an unramified extension of $\mathbb{Z}_{p}, p \geq 3$, of degree $f$. Then we have

$$
\begin{aligned}
\mathrm{K}_{2 n}\left(\mathcal{O}_{\mathfrak{p}}, \mathbb{Z}_{p}\right) & =\pi_{2 n-1}(\operatorname{im} J)_{p} \\
\mathrm{~K}_{2 n-1}\left(\mathcal{O}_{\mathfrak{p}}, \mathbb{Z}_{p}\right) & =\mathbb{Z}_{p}^{f} \oplus \pi_{2 n-1}(\operatorname{im} J)_{p} .
\end{aligned}
$$

Here $\operatorname{im} J$ is the image of the $J$-spectrum, i.e. $\pi_{4 n-1}(\operatorname{im} J)_{p}=\left(\mathbb{Z} / d_{n}\right)_{p}$, where $d_{n}$ is the denominator of the Bernoulli-number $\frac{B_{n}}{n}$.

For $\mathrm{K}_{3}$, the last theorem has also been proven by Levine [13].

Let us compare the last theorem with the Lichtenbaum-Quillen conjectures:

By the localization sequence we have for $K_{\mathfrak{p}}$ the quotient field of $\mathcal{O}_{\mathfrak{p}}$ and $n \geq 2$ : $\mathrm{K}_{n}\left(\mathcal{O}_{\mathfrak{p}}, \mathbb{Z}_{p}\right)=\mathrm{K}_{n}\left(K_{\mathfrak{p}}, \mathbb{Z}_{p}\right)$. One formulation of the Lichtenbaum-Quillen conjectures in this case is that the natural surjection [6]

$$
\rho: \mathrm{K}_{i}\left(K_{\mathfrak{p}}, \mathbb{Z}_{p}\right) \rightarrow \mathrm{K}_{i}^{e t}\left(K_{\mathfrak{p}}\right)
$$

is an isomorphism for sufficiently large $i$. By the splitting of the Dwyer-Friedlander spectral sequence for $\mathrm{K}_{*}^{e t}$, [20, theorem 1], we have

$$
\begin{aligned}
\mathrm{K}_{2 n}^{e t}\left(K_{\mathfrak{p}}\right) & =H^{0}\left(K_{\mathfrak{p}}, \mathbb{Z}_{p}(n)\right) \oplus H^{2}\left(K_{\mathfrak{p}}, \mathbb{Z}_{p}(n+1)\right) \\
\mathrm{K}_{2 n-1}^{e t}\left(K_{\mathfrak{p}}\right) & =H^{1}\left(K_{\mathfrak{p}}, \mathbb{Z}_{p}(n)\right) .
\end{aligned}
$$

The right hand side has been calculated in [17, par. 3], and we get with $w_{n}\left(K_{\mathfrak{p}}\right)=$ $\max \left\{p^{j}:\left[K_{\mathfrak{p}}\left(\mu_{p^{j}}\right): K_{\mathfrak{p}}\right] \mid n\right\}$.

Conjecture 3.3. (Lichtenbaum-Quillen conjecture for local fields)

$$
\begin{aligned}
\mathrm{K}_{2 n}\left(\mathcal{O}_{\mathfrak{p}}, \mathbb{Z}_{p}\right) & =\mathbb{Z} / w_{n}\left(K_{\mathfrak{p}}\right) \\
\mathrm{K}_{2 n-1}\left(\mathcal{O}_{\mathfrak{p}}, \mathbb{Z}_{p}\right) & =\mathbb{Z}_{p}^{f} \oplus \mathbb{Z} / w_{n}\left(K_{\mathfrak{p}}\right) .
\end{aligned}
$$


For $K_{\mathfrak{p}}$ unramified we have $w_{n}\left(K_{\mathfrak{p}}\right)=\# \pi_{2 n-1}(\operatorname{im} J)_{p}$, in particular we see that the surjections $\rho$ must be isomorphisms.

We also have an action of Adams operators on both the K-groups and on the constituents of the Dwyer-Friedlander spectral sequence. The Adams operator $\psi^{k}$ acts like $k^{n}$ on $H^{i}\left(K_{\mathfrak{p}}, \mathbb{Z}_{p}(n)\right)=E_{2}^{i,-2 n}=E_{\infty}^{i,-2 n}$, see [20, prop. 2, theorem 1], so we get:

Proposition 3.4. Let $\mathcal{O}_{\mathfrak{p}}$ be an unramified extension of $\mathbb{Z}_{p}$ and $p \geq 3$. Then we have

$$
\begin{aligned}
\mathrm{K}_{2 n}\left(\mathcal{O}_{\mathfrak{p}}, \mathbb{Z}_{p}\right) & =\mathrm{K}_{2 n}\left(\mathcal{O}_{\mathfrak{p}}, \mathbb{Z}_{p}\right)^{(n+1)} \\
\mathrm{K}_{2 n-1}\left(\mathcal{O}_{\mathfrak{p}}, \mathbb{Z}_{p}\right) & =\mathrm{K}_{2 n-1}\left(\mathcal{O}_{\mathfrak{p}}, \mathbb{Z}_{p}\right)^{(n)}
\end{aligned}
$$

\section{THE COKERNEL OF $K_{3}\left(\mathcal{O}_{\mathfrak{p}} / \mathfrak{p}^{r}\right) \rightarrow K_{3}\left(\mathcal{O}_{\mathfrak{p}} / \mathfrak{p}^{n}\right)$}

Let $\mathcal{O}_{\mathfrak{p}}$ be a finite extension of $\mathbb{Z}_{p}$ with ramification index $e$ and residue degree $f$. We will examine the cokernel of the maps $K_{3}\left(\mathcal{O}_{\mathfrak{p}} / \mathfrak{p}^{r}\right) \rightarrow \mathrm{K}_{3}\left(\mathcal{O}_{\mathfrak{p}} / \mathfrak{p}^{n}\right)$. We assume for simplicity $\mathrm{K}_{2}\left(\mathcal{O}_{\mathfrak{p}} / \mathfrak{p}^{r}\right)=0$, which is for example true in case $\mathcal{O}_{\mathfrak{p}}$ does not contain $p$-th roots of unity or if $r<\frac{p}{p-1} e,[5$, theorem 5.1]. This implies that $\mathrm{K}_{3}\left(\mathcal{O}_{\mathfrak{p}} / \mathfrak{p}^{r}\right)=H_{3}\left(S L\left(\mathcal{O}_{\mathfrak{p}} / \mathfrak{p}^{r}\right)\right)$ and similarly for $\mathrm{K}_{3}\left(\mathcal{O}_{\mathfrak{p}} / \mathfrak{p}^{n}\right)$.

Proposition 4.1. Suppose that $n \leq r \leq 2 n$ and $\mathrm{K}_{2}\left(\mathcal{O}_{\mathfrak{p}} / \mathfrak{p}^{r}\right)=0$. Then we have an exact sequence

$$
\mathrm{K}_{3}\left(\mathcal{O}_{\mathfrak{p}} / \mathfrak{p}^{r}\right) \rightarrow \mathrm{K}_{3}\left(\mathcal{O}_{\mathfrak{p}} / \mathfrak{p}^{n}\right) \rightarrow \mathcal{O}_{\mathfrak{p}} / \mathfrak{p}^{c} \rightarrow 0
$$

where $c=\min \left(r-n, d,(n-1)+v_{\mathfrak{p}}(n)\right)$, d the exponent of the discriminant of $\mathcal{O}_{\mathfrak{p}}$.

Proof: Consider the spectral sequence of homology groups associated to the short exact sequence

$$
0 \rightarrow N_{n}^{r} \rightarrow S L\left(\mathcal{O}_{\mathfrak{p}} / \mathfrak{p}^{r}\right) \rightarrow S L\left(\mathcal{O}_{\mathfrak{p}} / \mathfrak{p}^{n}\right) \rightarrow 0
$$

Since $r \leq 2 n$, the map $A \mapsto 1+A$ induces an isomorphism between $V\left(\mathfrak{p}^{n} / \mathfrak{p}^{r}\right)$, the trace zero matrices with entries in $\mathfrak{p}^{n} / \mathfrak{p}^{r}$, and $N_{n}^{r}$. Thus $H_{1}\left(N_{n}^{r}\right)=V\left(\mathfrak{p}^{n} / \mathfrak{p}^{r}\right)$ and we have $H_{2}\left(N_{n}^{r}\right)=\Lambda^{2} V\left(\mathfrak{p}^{n} / \mathfrak{p}^{r}\right)$, [3, theorem 6.4]. This gives us

$$
\begin{aligned}
& E_{1,0}^{2}=H_{1}\left(S L\left(\mathcal{O}_{\mathfrak{p}} / \mathfrak{p}^{n}\right)\right)=S L\left(\mathcal{O}_{\mathfrak{p}} / \mathfrak{p}^{n}\right)^{a b}=0 \\
& E_{2,0}^{2}=H_{2}\left(S L\left(\mathcal{O}_{\mathfrak{p}} / \mathfrak{p}^{n}\right)\right)=\mathrm{K}_{2}\left(\mathcal{O}_{\mathfrak{p}} / \mathfrak{p}^{n}\right)=0 \\
& E_{3,0}^{2}=H_{3}\left(S L\left(\mathcal{O}_{\mathfrak{p}} / \mathfrak{p}^{n}\right)\right)=\mathrm{K}_{3}\left(\mathcal{O}_{\mathfrak{p}} / \mathfrak{p}^{n}\right) \\
& E_{0,1}^{2}=H_{0}\left(S L\left(\mathcal{O}_{\mathfrak{p}} / \mathfrak{p}^{n}\right), V\left(\mathfrak{p}^{n} / \mathfrak{p}^{r}\right)\right)=0 \quad[12, \text { prop. 1.2] } \\
& E_{0,2}^{2}=H_{0}\left(S L\left(\mathcal{O}_{\mathfrak{p}} / \mathfrak{p}^{n}\right), \Lambda^{2} V\left(\mathfrak{p}^{n} / \mathfrak{p}^{r}\right)\right)=0 \quad[12, \text { théorème 3.4] } \\
& \qquad \begin{array}{l|c|c|c|c}
2 & 0 & & & \\
\hline 1 & 0 & E_{1,1}^{2} & & \\
\hline 0 & \mathbb{Z} & 0 & 0 & \mathrm{~K}_{3}\left(\mathcal{O}_{\mathfrak{p}} / \mathfrak{p}^{n}\right) \\
\hline & 0 & 1 & 2 & 3
\end{array}
\end{aligned}
$$

So we get the exact sequence

$$
\mathrm{K}_{3}\left(\mathcal{O}_{\mathfrak{p}} / \mathfrak{p}^{r}\right) \rightarrow \mathrm{K}_{3}\left(\mathcal{O}_{\mathfrak{p}} / \mathfrak{p}^{n}\right) \stackrel{d_{3,0}^{2}}{\longrightarrow} E_{1,1}^{2} \longrightarrow 0
$$

By [12, théorème 2.16] we have:

$$
E_{1,1}^{2}=H_{1}\left(S L\left(\mathcal{O}_{\mathfrak{p}} / \mathfrak{p}^{n}\right), V\left(\mathfrak{p}^{n} / \mathfrak{p}^{r}\right)\right)=\Omega_{\mathcal{O}_{\mathfrak{p}} / \mathfrak{p}^{n}} \otimes_{\mathcal{O}_{\mathfrak{p}} / \mathfrak{p}^{n}} \mathfrak{p}^{n} / \mathfrak{p}^{r}
$$


Since $\Omega_{\mathcal{O}_{\mathfrak{p}}}=\mathcal{O}_{\mathfrak{p}} / \mathfrak{p}^{d} d \pi$ for $\pi$ a uniformizer of $\mathcal{O}_{\mathfrak{p}}, d$ the valuation of the discriminant, and $e-1 \leq d \leq e-1+v_{\mathfrak{p}}(e)$, [18, p. 58-59], we have the exact sequence

$$
\mathfrak{p}^{n} / \mathfrak{p}^{2 n} \stackrel{\delta}{\rightarrow} \Omega_{\mathcal{O}_{\mathfrak{p}}} \otimes_{\mathcal{O}_{\mathfrak{p}}} \mathcal{O}_{\mathfrak{p}} / \mathfrak{p}^{n} \rightarrow \Omega_{\mathcal{O}_{\mathfrak{p}} / \mathfrak{p}^{n}} \rightarrow 0,
$$

where $\delta(x)=d x \otimes 1$. From $d \pi^{n}=n \pi^{n-1} d \pi$ we get

$$
\Omega_{\mathcal{O}_{\mathfrak{p}} / \mathfrak{p}^{n}}=\frac{\mathcal{O}_{\mathfrak{p}} / \mathfrak{p}^{d} d \pi \otimes_{\mathcal{O}_{\mathfrak{p}}} \mathcal{O}_{\mathfrak{p}} / \mathfrak{p}^{n}}{\mathcal{O}_{\mathfrak{p}} d \pi^{n} \otimes 1}=\mathcal{O}_{\mathfrak{p}} / \mathfrak{p}^{\min \left(n, d,(n-1)+v_{\mathfrak{p}}(n)\right)}
$$

Corollary 4.2. If $\mathcal{O}_{\mathfrak{p}}$ is unramified, then $\mathrm{K}_{3}\left(\mathcal{O}_{\mathfrak{p}} / \mathfrak{p}^{r}\right) \rightarrow \mathrm{K}_{3}\left(\mathcal{O}_{\mathfrak{p}} / \mathfrak{p}^{n}\right)$ is surjective for all $r>n$. Consequently $\mathrm{K}_{3}\left(\mathcal{O}_{\mathfrak{p}}, \mathbb{Z}_{p}\right)$ surjects onto $\mathrm{K}_{3}\left(\mathcal{O}_{\mathfrak{p}} / \mathfrak{p}^{n}\right)_{p}$.

More generally, for $r$ not necessarily less than or equal to $2 n$, the term $E_{0,2}^{2} / \mathrm{im} d_{2,1}^{2}=$ $H_{0}\left(S L\left(\mathcal{O}_{\mathfrak{p}} / \mathfrak{p}^{n}\right), H_{2}\left(V\left(\mathfrak{p}^{n} / \mathfrak{p}^{r}\right)\right)\right) / \operatorname{im} d_{2,1}^{2}$ gives an extra contribution to the cokernel. For example for $e>r$, the cokernels grow regularly by $(\mathbb{Z} / p)^{f}$ for $r=n+$ $1, \ldots, \min \left(2 n, 2 n-1+v_{\mathfrak{p}}(n)\right)$ (because $\left.\mathcal{O}_{\mathfrak{p}} / \mathfrak{p}^{c}=\mathbb{F}_{q}^{c}\right)$ until they reach $\mathbb{F}_{q}^{\min \left(n, n-1+v_{\mathfrak{p}}(n)\right)}$ and the $E_{1,1}$-contribution is exhausted. Then there is an irregular contribution coming from $E_{0,2} / \operatorname{im} d_{2,1}^{2}$. In case $\mathcal{O}_{\mathfrak{p}}$ sufficiently ramified (i.e. $\left.e>r\right), \mathrm{K}_{3}\left(\mathcal{O}_{\mathfrak{p}} / \mathfrak{p}^{n}\right)_{p}$ eventually equals the cokernel, the precise pattern can be read of from $[11,3.4]$.

For example the cokernel grows for the following $r$ :

$$
\begin{aligned}
p=3, \quad n=5: \quad 6,7,8,9,12,18,27,81 \\
p=3, \quad n=9: \quad 10,11,12,13,14,15,16,17,18,21,24,27,36,45,54,81 \\
p=5, \quad n=5: \quad 6,7,8,9,10,15,20,25 \\
p=5, \quad n=6: \quad 7,8,9,10,11,15,20,25,50,125
\end{aligned}
$$

\section{The OUTER AUTOMORPHISM}

The outer automorphism

$$
\begin{aligned}
\tau: S L(R) & \rightarrow S L(R) \\
A & \mapsto{ }^{t} A^{-1}
\end{aligned}
$$

induces an automorphism of order 2 on homology groups with coefficients in any self-dual representation. For $R=\mathbb{F}_{q}$ and as coefficients the homology groups of the adjoint representation $V$, the automorphism is compatible with the stabilization maps $S L_{n}(R) \rightarrow S L_{n+1}(R)$.

For the extension $1 \rightarrow V\left(\mathbb{F}_{q}\right) \rightarrow S L\left(W_{2}\left(\mathbb{F}_{q}\right)\right) \rightarrow S L\left(\mathbb{F}_{q}\right) \rightarrow 1$ the induced action on $V$ is given by $A \mapsto-{ }^{t} A$. The automorphism induces a map on the spectral sequences, all terms of the spectral sequence decompose into +- and --eigenspaces and the differentials respect this decomposition.

We will determine the action of $\tau$ on some of the $E_{2}$-terms:

Proposition 5.1. a) The automorphism $\tau$ acts like +1 on $H_{0}\left(S L\left(\mathbb{F}_{q}\right), \Lambda^{3} V\right)$ and on $H_{0}\left(S L\left(\mathbb{F}_{q}\right), S^{2} V\right)$.

b) For $n \geq 2$ and $p \geq n, \tau$ acts like $(-1)^{n}$ on $H_{2 n-2}\left(S L\left(\mathbb{F}_{q}\right), V\right)=\mathbb{F}_{q}$. 
Proof: a) We prove the dual cohomological result. The stabilization maps

$$
H^{0}\left(S L_{n}\left(\mathbb{F}_{q}\right), \Lambda^{3} V_{n}^{*}\right) \rightarrow H^{0}\left(S L_{2}\left(\mathbb{F}_{q}\right), \Lambda^{3} V_{2}^{*}\right)
$$

are isomorphisms, as one sees from the diagram

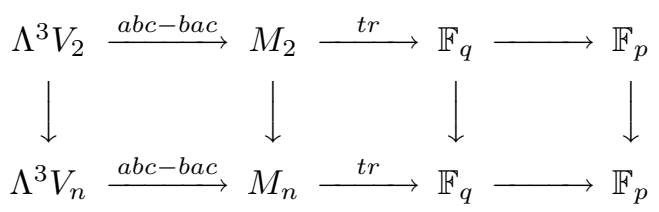

and similarly for $S^{2} V^{*}$. But on the $S L_{2}$-level $\tau$ is an inner automorphism, thus the action must be trivial.

b) By $[19$, theorem 7.6] we can always go to a bigger field and thus assume that $2 n-2<f(2 p-3)-2$. By duality and lemma 2.1 we have

$$
H_{2 n-2}\left(S L\left(\mathbb{F}_{q}\right), V\right)=H^{2 n-2}\left(S L\left(\mathbb{F}_{q}\right), V^{*}\right)^{*}=H^{2 n-2}\left(G L\left(\mathbb{F}_{q}\right), M^{*}\right)^{*}
$$

and since we assume $2 n-2<\min (2 p-1, f(2 p-3)-2)$ we know by [8] that we have stably

$$
H^{2 n-2}\left(G L\left(\mathbb{F}_{q}\right), M\right)=H^{2 n-2}\left(B_{n}\left(\mathbb{F}_{q}\right), M_{n}\right)=\mathbb{F}_{q},
$$

where $B_{n}\left(\mathbb{F}_{q}\right)$ is the Borel subgroup of upper triangular matrices.

Instead of $\tau$ we consider the composition $\sigma$ of $\tau$ with conjugation by $g$, where $g=\left(a_{i, j}\right)$ with $a_{i, j}=1$ for $j+i=n+1$ and 0 otherwise, because $\sigma$ respects the Borel subgroup.

An easy calculation shows that $\sigma$ acts on $M_{n}=\operatorname{ker} G L_{n}\left(W_{2}\left(\mathbb{F}_{q}\right)\right) \rightarrow G L_{n}\left(\mathbb{F}_{q}\right)$ by $\left(a_{i, j}\right) \rightarrow\left(-a_{n+1-j, n+1-i}\right)$ (i.e. -1 times the reflection on the diagonal $\left.(1, n) \ldots(n, 1)\right)$, since $\tau\left(a_{i, j}\right)=-\left(a_{i, j}\right)^{t}$ and Int $g$ induces a turn by 180 degree.

We define the following $\sigma$-invariant descending filtration on $M_{n}$ :

$$
F^{s} M_{n}=\left\{\left(a_{i, j}\right) \mid a_{i, j}=0 \text { for } i-j \geq n-s\right\} .
$$

The associated graded pieces are isomorphic to

$$
\operatorname{gr}^{s} M_{n}=\left\{\left(a_{i, j}\right) \mid a_{i, j}=0 \quad \text { for } \quad i-j \neq n-s-1\right\} .
$$

\section{Lemma 5.2.}

$$
H^{2 n-2}\left(B_{n}, \operatorname{gr}^{s} M_{n}\right)= \begin{cases}\mathbb{F}_{q} & \text { for } s=2 n-2 \\ 0 & \text { otherwise }\end{cases}
$$

Proof: To compute the cohomology of $B_{n}$ with coefficients in the graded pieces we use the "symbolic weight equations" of [8]:

First note that for $U_{n}$ the unipotent subgroup of $B_{n}$ and $T_{n}$ its torus, we have

$$
H^{2 n-2}\left(B_{n}, \mathrm{gr}^{s} M_{n}\right)=H^{2 n-2}\left(U_{n}, \mathrm{gr}^{s} M_{n}\right)^{T_{n}}=\left(H^{2 n-2}\left(U_{n}, \mathbb{F}_{q}\right) \otimes_{\mathbb{F}_{q}} \mathrm{gr}^{s} M_{n}\right)^{T_{n}} .
$$

The first equation follows because the order of $T_{n}$ is prime to $p$ and $\mathrm{gr}^{s} M_{n}$ is a $p$-torsion group. The second equation follows because $U_{n}$ acts trivially on $\operatorname{gr}^{s} M_{n}$.

In [8] one sees that $U_{n}$ admits a filtration such that we have for the graded pieces $\operatorname{gr} U_{n}=\mathbb{F}_{q}^{n(n-1) / 2}$ and for the cohomology $H^{2 n-2}\left(U_{n}, \mathbb{F}_{q}\right)=H^{2 n-2}\left(\operatorname{gr} U_{n}, \mathbb{F}_{q}\right)$. On the other hand the cohomology of gr $U_{n}$ is given by

$$
H^{*}\left(\operatorname{gr} U_{n}, \mathbb{F}_{q}\right)=\Lambda_{\mathbb{F}_{q}}^{*}\left(V_{n}\right) \otimes_{\mathbb{F}_{q}} S_{\mathbb{F}_{q}}^{*}\left(W_{n}\right),
$$

where $V_{n}$ has a basis $\left\{a_{i, j}^{s} \mid 1 \leq i<j \leq n, 0 \leq s<f\right\}$ and is of degree 1 , and $W_{n}$ has a basis $\left\{b_{i, j}^{s} \mid 1 \leq i<j \leq n, 0 \leq s<f\right\}$ and is of degree 2. The $T_{n}$-action on 
this ring is given by the condition that $a_{i, j}^{s}$ and $b_{i, j}^{s}$ have weight $-p^{s} \alpha_{i, j}$, where $\alpha_{i, j}$ is the character $\left(t_{1}, \ldots, t_{n}\right) \mapsto t_{i} / t_{j}$. We write this symbolically as

$$
\left[a_{i, j}^{s}\right]=\left[b_{i, j}^{s}\right]=-p^{s}[i]+p^{s}[j] .
$$

The $T_{n}$-action on $e_{u, v} \in \operatorname{gr}^{s} M_{n}(u-v=n-s-1)$ is given by $\alpha_{u, v}$, so it has symbolic weight $[u]-[v]$. We want to determine

$$
\left(H^{2 n-2}\left(\operatorname{gr} U_{n}, \mathbb{F}_{q}\right) \otimes_{\mathbb{F}_{q}} \operatorname{gr}^{s} M_{n}\right)^{T_{n}} .
$$

As $T_{n}$ acts like scalars on all basis elements of $H^{2 n-2}\left(\operatorname{gr} U_{n}, \mathbb{F}_{q}\right)$ and $\operatorname{gr}^{s} M_{n}$, it suffices to consider monomials of the form

$$
z=a_{i_{1}, j_{1}}^{s_{1}} \wedge \cdots \wedge a_{i_{m}, j_{m}}^{s_{m}} \otimes b_{k_{1}, l_{1}}^{t_{1}} \otimes \cdots \otimes b_{k_{r}, l_{r}}^{t_{r}} \otimes e_{u, v} \in \Lambda_{\mathbb{F}_{q}}^{m} V_{n} \otimes S_{\mathbb{F}_{q}}^{r} W_{n} \otimes \operatorname{gr}^{s} M_{n}
$$

for $m+2 r=2 n-2$ and $u-v=n-s-1$ in order to get all $T_{n}$-invariant elements. The monomial $z$ has symbolic weight

$$
[z]=-p^{s_{1}}\left[i_{1}\right]+p^{s_{1}}\left[j_{1}\right]-\cdots+p^{t_{r}}\left[l_{r}\right]+[u]-[v]=: \sum_{e=1}^{n} g_{e}[e] .
$$

Obviously the sum of the positive $g_{e}$ equals minus the sum of the negative $g_{e}$. In order for $z$ to be $T_{n}$-invariant, we need $g_{e} \equiv 0 \bmod p^{f}-1$ for all $e$.

Let $l_{1}$ be the smallest subscript occurring. If $g_{l_{1}}=0$, we must have $u=l_{1}$ and the only $a$ and $b$ occurring with $l_{1}$ as the first subscript is $a_{l_{1}, k_{1}}^{0}$ or $b_{l_{1}, k_{1}}^{0}$ for some $k_{1}$. In this case let $i_{2}$ be the next smallest subscript occurring. Again, if $g_{l_{2}}=0$, then $l_{2}=k_{1}$ and there is at most one $a_{l_{2}, k_{2}}^{0}$ or $b_{l_{2}, k_{2}}^{0}$ occurring for some $k_{2}$. Continuing in this fashion, we either find a smallest $l$ such that $g_{l} \neq 0$, and all but one coefficients of $[l]$ are negative (and the positive coefficient can only be 1 ), or all $g_{e}=0$ and $z$ is made from elements $c_{l_{1}, l_{2}}^{0}, c_{l_{2}, l_{3}}^{0}, \cdots, c_{l_{m}, l_{m+1}}^{0}$ with $u=l_{1}<l_{2} \cdots<l_{m+1}=v$. Clearly $m+1 \leq l_{m+1} \leq n$, on the other hand $\operatorname{deg} z=2 n-2 \leq 2 m$, so we conclude $m=n-1, l_{i}=i$ and

$$
z=b_{1,2}^{0} \otimes \cdots \otimes b_{n-1, n}^{0} \otimes e_{1, n} .
$$

Thus we find a unique basic element in $H^{2 n-2}\left(B_{n}, \operatorname{gr}^{s} M\right)$ for $s=2 n-2$.

In case there is a smallest $l$ such that $g_{l} \neq 0$ we similarly find a largest $j$ such that $g_{j} \neq 0$, and all but one coefficients of $j$ are positive (and the one exception can only be -1 ).

Consider the minimal $p$-adic expression

$$
\left|g_{e}\right|=\sum_{\nu=0}^{f-1} g_{e, \nu} p^{\nu}
$$

where minimal means that $\sum g_{e, \nu}$ is minimal. We have

$$
-g_{l}=\sum_{\nu=0}^{f-1} g_{l, \nu} p^{\nu} \equiv 0 \quad \bmod p^{f}-1
$$

Because $g_{l} \neq 0$ and $z$ has $2 n-2$ factors with coefficients at most $p^{f-1}$, we have $-g_{l} \leq(2 n-2) p^{f-1} \leq(2 p-2) p^{f-1}<2\left(p^{f}-1\right)$. So $-g_{l}=p^{f}-1$ and we can conclude $\sum g_{l, \nu} \geq f(p-1)$. Similarly we get $\sum g_{j, \nu} \geq f(p-1)$.

Let $I$ be the number of factors of $z$ of the form $a_{l, j}^{s}$ and $b_{l, j}^{s}$, then the sum of degrees of these terms is $f+2(I-f)$, as there are at most $f$ factors of this form 
of cohomological degree 1 . Since the number of factors with an $l$ occurring as a subscript is at least $\sum g_{l, \nu}$, we get

$$
2 n-2=\operatorname{deg} z \geq\left(\sum g_{l, \nu}-I\right)+\left(\sum g_{j, \nu}-I\right)+(f+2(I-f)) \geq f(2 p-3),
$$

contradicting $2 n-2<f(2 p-3)-2$.

We now consider the spectral sequence to the filtration $F^{s} M_{n}$,

$$
E_{1}^{s, t}=H^{s+t}\left(B_{n}, \operatorname{gr}^{s} M_{n}\right) \Rightarrow H^{s+t}\left(B_{n}, M_{n}\right)
$$

From

we conclude that we have

$$
H^{2 n-2}\left(B_{n}, \mathrm{gr}^{s} M_{n}\right)= \begin{cases}\mathbb{F}_{q} & \text { for } \quad s=2 n-2 \\ 0 & \text { otherwise }\end{cases}
$$

$$
H^{2 n-2}\left(B_{n}, \operatorname{gr}^{2 n-2} M_{n}\right)=E_{1}^{2 n-2,0}=E_{\infty}^{2 n-2,0}=H^{2 n-2}\left(B_{n}, M_{n}\right)=\mathbb{F}_{q}
$$

and we can calculate the action of $\sigma$ on $H^{2 n-2}\left(B_{n}, \operatorname{gr}^{s} M_{n}\right)$.

But as $H^{2 n-2}\left(B_{n}, M_{n}\right)=H^{2 n-2}\left(B_{n}, \mathbb{F}_{q} e_{1, n}\right)$ is generated by the cocycle

$$
z=b_{1,2}^{0} \otimes \cdots \otimes b_{n-1, n}^{0} \otimes e_{1, n}
$$

we have to calculate the action of $\sigma$ on $z$. An easy calculation shows that $\sigma\left(e_{1, n}\right)=$ $-e_{1, n}$ and $\sigma\left(b_{i, j}\right)=-b_{n+1-j, n+1-i}$. As the $b_{i, j}$ commute we get $\sigma(z)=(-1)^{n-1+1} z$, which was to be proven.

We are now ready to determine $\mathrm{K}_{3}\left(W_{2}\left(\mathbb{F}_{q}\right)\right)$ in characteristic at least 5 .

Proposition 5.3. For $p \geq 3$ we have the following + -eigenspaces under $\tau$ in the spectral sequence $H_{i}\left(S L\left(\mathbb{F}_{p^{f}}\right), H_{j}(V)\right)_{p} \Rightarrow H_{i+j}\left(S L\left(W_{2}\left(\mathbb{F}_{p^{f}}\right)\right)\right)_{p}$ :

\begin{tabular}{c|c|c|c|c|c}
3 & $(\mathbb{Z} / p)^{2 f}$ & & & & \\
\hline 2 & 0 & 0 & $(\mathbb{Z} / p)^{f}$ & & \\
\hline 1 & 0 & 0 & $(\mathbb{Z} / p)^{f}$ & 0 & 0 \\
\hline 0 & $\mathbb{Z}$ & 0 & 0 & 0 & 0 \\
\hline & 0 & 1 & 2 & 3 & 4
\end{tabular}

Proof: This is an immediate consequence of 2.3 and 5.1 except from the identity $\left(E_{2,2}^{2}\right)^{+}=H^{+}=(\mathbb{Z} / p)^{f}$. For this consider the extension

$$
0 \rightarrow V \rightarrow S L\left(\mathbb{F}_{p^{f}}[t] / t^{2}\right) \rightarrow S L\left(\mathbb{F}_{p^{f}}\right) \rightarrow 0
$$

The corresponding spectral sequence has the same $E_{2}$-terms as the spectral sequence to the extension $0 \rightarrow V \rightarrow S L\left(\mathrm{~W}_{2}\left(\mathbb{F}_{\mathrm{q}}\right)\right) \rightarrow S L\left(\mathbb{F}_{p^{f}}\right) \rightarrow 0$, since the action of $S L\left(\mathbb{F}_{p^{f}}\right)$ on $V$ is the adjoint action in both cases. The differentials are different, however, as the latter sequence does not split whereas the former does.

From $\# \mathrm{~K}_{3}\left(\mathbb{F}_{p^{f}}[t] / t^{2}\right)=p^{2 f}$ we conclude that $E_{0,3}^{\infty}=(\mathbb{Z} / p)^{f}$ and thus that $d_{2,2}^{2}$ has rank $f$. On the other hand we know that $\mathrm{K}_{4}\left(\mathbb{F}_{p^{f}}[t] / t^{2}\right)=0$, so $E_{2,2}^{\infty}=0$. As there are no nonzero differentials ending in $E_{2,2}$, we conclude $H^{+}=(\mathbb{Z} / p)^{f}$.

Proof: of theorem $1.1 \mathrm{a})$ :

By Suslins result the prime to $p$-part is the same as for $\mathbb{F}_{q}$. For the $p$-part let us first determine the +-eigenspaces. By 4.2 and 3.2 we know that $\mathrm{K}_{3}\left(W_{2}\left(\mathbb{F}_{p^{f}}\right)\right)_{p}$ has at most $f$ generators. This forces the differential $d_{2,2}^{2}$ in 5.3 to be injective. Thus 
we are left with a group with $f$ generators and two graded pieces isomorphic to $(\mathbb{Z} / p)^{f}$, giving the desired result for the +-eigenspaces.

As $\mathrm{K}_{3}\left(W_{2}\left(\mathbb{F}_{p^{f}}\right)\right)_{p}$ has at most $f$ generators and the +-eigenspace already has $f$ generators, we conclude that the --eigenspace is trivial.

\section{6. $K_{3}\left(W_{2}\left(\mathbb{F}_{q}\right)\right)$ FOR CHAR $\mathbb{F}_{q}=3$}

In this section we determine $\mathrm{K}_{3}\left(W_{2}\left(\mathbb{F}_{3^{f}}\right)\right)$ for $(3, f)=1$. The problem in characteristic 3 is that $K_{3}\left(W_{2}\left(\mathbb{F}_{3 f}\right)\right)_{3}$ might have $f+1$ generators instead of $f$ generators and so the differential $d_{2,2}^{2}$ in 5.3 may not be injective (and similar in the mod 3 spectral sequence).

It turns out that cohomological calculations are easier than homological calculations, so from now on we work with cohomology groups. The dual of 5.3 gives us the following $E_{2}$-terms in the spectral sequences:

$$
\begin{aligned}
& \begin{array}{lc|c|c|c|c|c} 
& 3 & (\mathbb{Z} / 3)^{2 f} & & & & \\
\hline 2 & 0 & 0 & (\mathbb{Z} / 3)^{2 f} & & \\
\hline H^{i}\left(S L\left(\mathbb{F}_{3^{f}}\right), H^{j}(V, \mathbb{Z} / 3)\right): & 1 & 0 & 0 & (\mathbb{Z} / 3)^{f} & 0 & 0 \\
\hline 0 & \mathbb{Z} & 0 & 0 & 0 & 0 \\
\hline & & 0 & 1 & 2 & 3 & 4
\end{array} \\
& \begin{array}{c|c|c|c|c|c|c}
4 & (\mathbb{Z} / 3)^{2 f} & & & & & \\
\hline 3 & 0 & 0 & (\mathbb{Z} / 3)^{f} & & & \\
\hline 2 & 0 & 0 & (\mathbb{Z} / 3)^{f} & 0 & 0 & \\
\hline 1 & 0 & 0 & 0 & 0 & 0 & 0 \\
\hline 0 & \mathbb{Z} & 0 & 0 & 0 & 0 & 0 \\
\hline & 0 & 1 & 2 & 3 & 4 & 5
\end{array}
\end{aligned}
$$

In order to determine $H^{3}\left(S L\left(W_{2}\left(\mathbb{F}_{3^{f}}\right)\right), \mathbb{Z} / 3\right)$ and $H^{4}\left(S L\left(W_{2}\left(\mathbb{F}_{3^{f}}\right)\right)\right)_{3}$, we have to calculate the differentials

$$
\begin{aligned}
& d_{2}^{0,3}: H^{0}\left(S L\left(\mathbb{F}_{3^{f}}\right), H^{3}(V, \mathbb{Z} / 3)\right) \longrightarrow H^{2}\left(S L\left(\mathbb{F}_{3^{f}}\right), H^{2}(V, \mathbb{Z} / 3)\right) \\
& \cong \delta \\
& d_{2}^{0,4}: H^{0}\left(S L\left(\mathbb{F}_{3^{f}}\right), H^{4}(V)\right) \quad \longrightarrow \quad H^{2}\left(S L\left(\mathbb{F}_{3^{f}}\right), H^{3}(V)\right)
\end{aligned}
$$

The calculations will be similar to the calculations in [7, par.9-11]. The idea is to use stability to reduce to the $S L_{2}$-level first, and then make the calculations for a 3 -Sylow group. However, as we are in characteristic 3, the short exact sequence

$$
1 \rightarrow V_{2} \rightarrow S L_{2}(\mathbb{Z} / 9) \rightarrow S L_{2}(\mathbb{Z} / 3) \rightarrow 1
$$

splits. Thus we would have to work on the $S L_{3}$-level. Instead we make calculations for $W_{2}\left(\mathbb{F}_{9}\right)$ and deduce results for $\mathbb{F}_{3}$, because the 3 -Sylow group of $S L_{2}\left(\mathbb{F}_{9}\right)$ is abelian and has only rank 2 .

We choose a basis $\{1, z\}$ of $\mathbb{F}_{9}$ over $\mathbb{F}_{3}$ such that $z^{2}=-1$, and consider the following short exact sequence

$$
1 \rightarrow V_{2} \rightarrow U \rightarrow P \rightarrow 1
$$

where $U$ is the 3-Sylow subgroup of $S L_{2}\left(W_{2}\left(\mathbb{F}_{9}\right)\right)$ consisting of matrices

$$
\left(\begin{array}{cc}
1+3 a & b \\
3 c & 1+3 d
\end{array}\right), \quad a+d-b c \equiv 0 \quad \bmod 3
$$


We get the following diagram

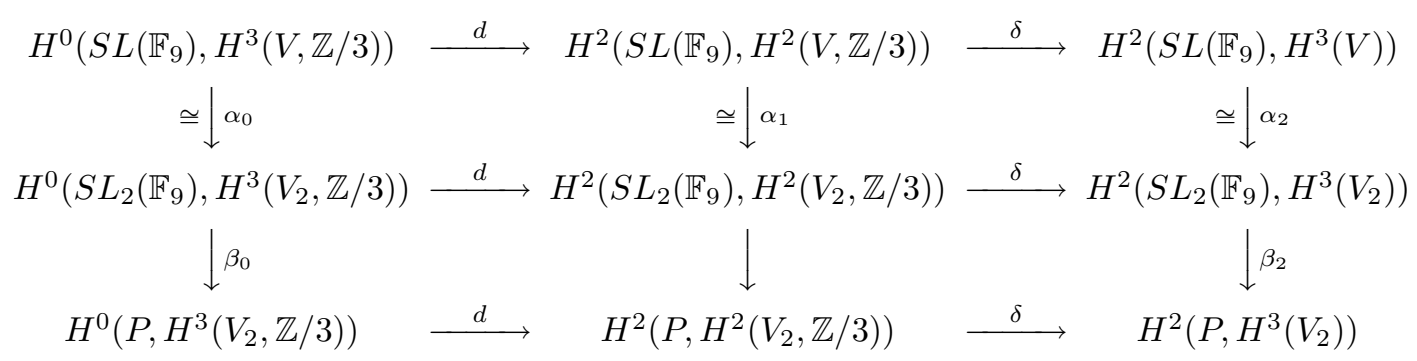

The map $\alpha_{0}$ is an isomorphism as in the proof of 5.1(a). To show that $\alpha_{1}$ and $\alpha_{2}$ are isomorphisms consider the following diagram:

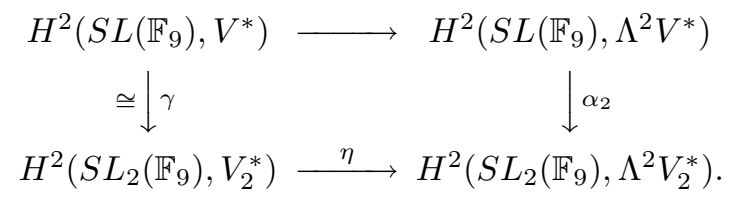

By [8, prop 1.6] and 2.1 the map $\gamma$ is an isomorphism. On the other hand the lower map $\eta$, induced by $a \wedge b \mapsto a b-b a$, is split by the map induced by $e_{i j} \mapsto \frac{1}{2} \sum_{k} e_{i k} \wedge e_{k j}$ and thus injective. Since all groups in the diagram equal $\mathbb{F}_{9}$, we see that $\alpha_{2}$ is an isomorphism. As $\alpha_{1}$ is the direct sum of $\alpha_{2}$ and $\gamma$, it must be an isomorphism too. The maps $\beta_{0}$ and $\beta_{2}$ are injective as $P$ is a 3-Sylow group of $S L_{2}\left(\mathbb{F}_{9}\right)$.

These considerations show that we can calculate the differential in the lower row of the image of $\beta_{0} \circ \alpha_{0}$.

From now on we will write $V$ for $V_{2}$, as there is no danger of confusion.

We have

$$
H^{0}\left(S L_{2}\left(\mathbb{F}_{9}\right), H^{3}(V, \mathbb{Z} / 3)\right)=\left(\Lambda^{3} V^{*}\right)^{S L_{2}\left(\mathbb{F}_{9}\right)} \oplus\left(S^{2} V^{*}\right)^{S L_{2}\left(\mathbb{F}_{9}\right)} .
$$

The invariants all have the form

$$
\begin{array}{lllllll}
\varphi: \Lambda^{3} V & \stackrel{a b c-b a c}{\longrightarrow} & M & \stackrel{t r}{\longrightarrow} & \mathbb{F}_{9} & \stackrel{\chi}{\longrightarrow} & \mathbb{F}_{3} \\
\psi: S^{2} V & \stackrel{a b}{\longrightarrow} & M & \stackrel{t r}{\longrightarrow} & \mathbb{F}_{9} & \stackrel{\chi}{\longrightarrow} & \mathbb{F}_{3},
\end{array}
$$

where $\chi$ runs through the linear forms. If we choose the linear forms $\chi_{1}: a+b z \mapsto a$ and $\chi_{2}: a+b z \mapsto b$ as a basis, we find the following basic invariant forms:

$$
A:=-\chi_{1} \circ \varphi, \quad B:=\chi_{2} \circ \varphi, \quad C:=\chi_{1} \circ \psi, \quad D:=\chi_{2} \circ \psi .
$$

Proposition 6.1. a)

$$
\mathrm{K}_{3}\left(W_{2}\left(\mathbb{F}_{9}\right)\right)=\mathbb{Z} / 9 \oplus \mathbb{Z} / 3 \oplus \mathbb{Z} / 3 \oplus \mathbb{Z} / 80
$$

b)

$$
\mathrm{K}_{3}(\mathbb{Z} / 9)=\mathbb{Z} / 3 \oplus \mathbb{Z} / 3 \oplus \mathbb{Z} / 8 .
$$

Proof: a) Consider the spectral sequence

$$
E_{2}^{i, j}(\mathbb{Z} / 3)=H^{i}\left(P, H^{j}(V, \mathbb{Z} / 3)\right) \Rightarrow H^{i+j}(U, \mathbb{Z} / 3)
$$

and its differential

$$
d_{2}^{0,3}: E_{2}^{0,3}(\mathbb{Z} / 3)=(\mathbb{Z} / 3)^{4} \rightarrow E_{2}^{2,2}(\mathbb{Z} / 3)=(\mathbb{Z} / 3)^{4} .
$$

We will see in 9.1 that $d_{2}^{0,3}(A+C)=0$, so $d_{2}^{0,3}$ has rank at most 3 . On the other hand it has rank at least 3 , because $K_{3}\left(W_{2}\left(\mathbb{F}_{q}\right)\right)$ has at most 3 generators by 4.2 and 3.2. So the number of generators of $K_{3}\left(W_{2}\left(\mathbb{F}_{9}\right)\right)$ is 3 . 
Now consider the spectral sequence

$$
E_{2}^{i, j}(\mathbb{Z})=H^{i}\left(P, H^{j}(V)\right) \Rightarrow H^{i+j}(U)
$$

with differential

$$
d_{2}^{0,4}: E_{2}^{0,4}(\mathbb{Z})=(\mathbb{Z} / 3)^{4} \rightarrow E_{2}^{2,3}(\mathbb{Z})=(\mathbb{Z} / 3)^{2}
$$

We will see in 9.1 that $d_{2}^{0,4}(A)$ and $d_{2}^{0,4}(B)$ are linearly independent, so $d_{2}^{0,4}(\mathbb{Z})$ has rank 2 , and the cardinality of $\mathrm{K}_{3}\left(W_{2}\left(\mathbb{F}_{9}\right)\right)_{3}$ is $3^{4}$.

b) The inclusion $i: \mathbb{Z} / 9 \rightarrow W_{2}\left(\mathbb{F}_{3^{f}}\right)$ induces the natural map $i_{*}: K_{3}(\mathbb{Z} / 9) \rightarrow$ $\mathrm{K}_{3}\left(W_{2}\left(\mathbb{F}_{3 f}\right)\right)$. On the other hand we have the transfer map $i^{*}: \mathrm{K}_{3}\left(W_{2}\left(\mathbb{F}_{3^{f}}\right)\right) \rightarrow$ $\mathrm{K}_{3}(\mathbb{Z} / 9)$ induced by considering a $W_{2}\left(\mathbb{F}_{3^{f}}\right)$-module as a $\mathbb{Z} / 9$-module. As $W_{2}\left(\mathbb{F}_{3^{f}}\right)$ is a free $\mathbb{Z} / 9$-module of rank $f$, we have that $i^{*} \circ i_{*}$ is multiplication by $f$.

Consider now the following diagram

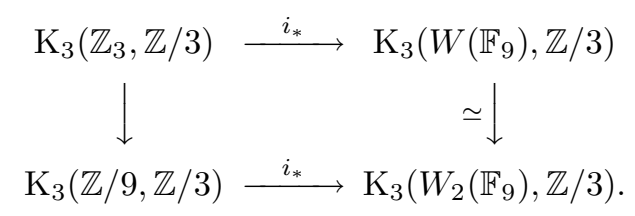

As the upper horizontal arrow is injective and the right vertical arrow is an isomorphism by a), the left vertical surjection must be an isomorphism and thus $K_{3}(\mathbb{Z} / 9)$ has 2 generators.

For the number of elements we use the following diagram:

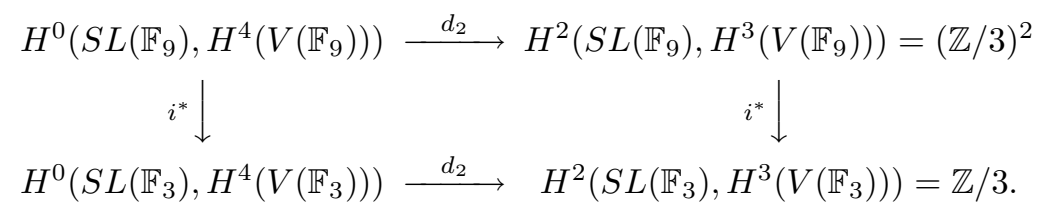

By the dual of [19, theorem 7.6] the vertical maps are surjective. And according to (a) the upper horizontal map is surjective, so the lower horizontal map must be surjective as well and thus the cardinality of $K_{3}(\mathbb{Z} / 9)_{3}$ is 9 .

Proof: of theorem $1.1 \mathrm{~b})$ : As in the above proposition we can conclude from $(3, f)=1$ that the natural map $i_{*} \operatorname{maps}(\mathbb{Z} / 3)^{2}=K_{3}(\mathbb{Z} / 9)_{3}$ to a direct summand of $K_{3}\left(W_{2}\left(\mathbb{F}_{3 f}\right)\right)_{3}$. We know by 5.3 that it is 9 -torsion and has at least $3^{2 f}$ elements. As it has at most $f+1$ generators by 4.2 and 3.2 , the theorem follows.

Remark: The result $K_{3}\left(\mathbb{Z}_{3}, \mathbb{Z}_{3}\right)=\mathbb{Z}_{3} \oplus \mathbb{Z} / 3$ of $[2]$ contradicts the results of $[1]$. Similarly, our result on $K_{3}(\mathbb{Z} / 9)$ contradicts the result $K_{3}(\mathbb{Z} / 9)_{3}=\mathbb{Z} / 9$ of $[1]$. The problem seems to be in [1, prop.II 4.5].

\section{Calculation of the differential $d_{2}^{0,3}$ in Characteristic 3}

Recall that we want to calculate a differential in a spectral sequence for the extension

$$
1 \rightarrow V \rightarrow U \rightarrow P \rightarrow 1
$$

where $U$ is the 3 -Sylow subgroup of $S L_{2}\left(W_{2}\left(\mathbb{F}_{9}\right)\right)$ such that $P$ consists of matrices of the form

$$
\left(\begin{array}{cc}
1 & x \\
0 & 1
\end{array}\right), \quad x \in \mathbb{F}_{9} .
$$


We choose for $P$ the basis

$$
t=\left(\begin{array}{cc}
1 & -1 \\
0 & 1
\end{array}\right), \quad s=\left(\begin{array}{cc}
1 & -z \\
0 & 1
\end{array}\right) .
$$

We also choose inverse images of $t$ and $s$ in $U$ of the same form. For $V$ we take as a basis the matrices (written multiplicatively)

$$
\begin{array}{rl}
x_{1}=\left(\begin{array}{ll}
1 & 0 \\
3 & 1
\end{array}\right) & \bar{x}_{1}=\left(\begin{array}{cc}
1 & 0 \\
3 z & 1
\end{array}\right) \\
x_{2}=\left(\begin{array}{cc}
1+3 & -3 \\
0 & 1-3
\end{array}\right) & \bar{x}_{2}=\left(\begin{array}{cc}
1+3 z & -3 z \\
0 & 1-3 z
\end{array}\right) \\
1 & 3 \\
x_{3}= & \bar{x}_{3}=\left(\begin{array}{cc}
1 & 3 z \\
0 & 1
\end{array}\right) .
\end{array}
$$

If we order this basis as $\left(x_{1}, \bar{x}_{1}, x_{2}, \bar{x}_{2}, x_{3}, \bar{x}_{3}\right)$, then the action of $t^{-1}$ and $s^{-1}$ is given by the matrices

$$
t^{-1}=\left(\begin{array}{cccccc}
1 & 0 & 0 & 0 & 0 & 0 \\
0 & 1 & 0 & 0 & 0 & 0 \\
1 & 0 & 1 & 0 & 0 & 0 \\
0 & 1 & 0 & 1 & 0 & 0 \\
0 & 0 & 1 & 0 & 1 & 0 \\
0 & 0 & 0 & 1 & 0 & 1
\end{array}\right), \quad s^{-1}=\left(\begin{array}{cccccc}
1 & 0 & 0 & 0 & 0 & 0 \\
0 & 1 & 0 & 0 & 0 & 0 \\
0 & -1 & 1 & 0 & 0 & 0 \\
1 & 0 & 0 & 1 & 0 & 0 \\
1 & -1 & 0 & -1 & 1 & 0 \\
1 & 1 & 1 & 0 & 0 & 1
\end{array}\right)
$$

for example the second column in $s^{-1}$ is obtained by

$s^{-1} \bar{x}_{1} s=\left(\begin{array}{ll}1 & z \\ 0 & 1\end{array}\right)\left(\begin{array}{rr}1 & 0 \\ 3 z & 1\end{array}\right)\left(\begin{array}{rr}1 & -z \\ 0 & 1\end{array}\right)=\left(\begin{array}{rr}1-3 & 3 z \\ 3 z & 1+3\end{array}\right)=\bar{x}_{1}-x_{2}-x_{3}+\bar{x}_{3}$.

If we denote the dual basis of $V^{*}$ by $\alpha_{1}, \bar{\alpha}_{1}, \alpha_{2}, \bar{\alpha}_{2}, \alpha_{3}, \bar{\alpha}_{3}$, then the action of $t$ and $s$ on $V^{*}$ is given by the transpose of the above matrices.

Proposition 7.1. The following are bases and dual bases for homology and cohomology groups of $V$ :

$$
\begin{array}{ccc}
H_{1}(V, \mathbb{Z} / p): & x_{i}, \bar{x}_{i} & \\
H^{1}(V, \mathbb{Z} / p): & \alpha_{i}, \bar{\alpha}_{i} & \\
H_{2}(V, \mathbb{Z} / p): & x_{i} \cap x_{j}, \bar{x}_{i} \cap \bar{x}_{j} & \\
& x_{i} \cap \bar{x}_{j} & \\
H^{2}(V, \mathbb{Z} / p): & \rho\left(x_{i}\right), \rho\left(\bar{x}_{i}\right) & \\
& \alpha_{i} \cup \alpha_{j}, \bar{\alpha}_{i} \cup \bar{\alpha}_{j} & \\
\alpha_{i} \cup \bar{\alpha}_{j} & \\
H_{3}(V, \mathbb{Z} / p): & \delta\left(\alpha_{i}\right), \delta\left(\bar{\alpha}_{i}\right) & \\
& x_{1} \cap x_{2} \cap x_{3}, \bar{x}_{1} \cap \bar{x}_{2} \cap \bar{x}_{3} & \\
& x_{i} \cap x_{j} \cap \overline{x_{k}}, \bar{x}_{i} \cap \bar{x}_{j} \cap x_{k} & i<j \\
H^{3}(V, \mathbb{Z} / p): & x_{i} \cap \rho\left(x_{j}\right), \bar{x}_{i} \cap \rho\left(x_{j}\right), x_{i} \cap \rho\left(\bar{x}_{j}\right), \bar{x}_{i} \cap \rho\left(\bar{x}_{j}\right) & \\
& \alpha_{1} \cup \alpha_{2} \cup \alpha_{3}, \bar{\alpha}_{1} \cup \bar{\alpha}_{2} \cup \bar{\alpha}_{3} & \\
& \alpha_{i} \cup \alpha_{j} \cup \bar{\alpha}_{k}, \bar{\alpha}_{i} \cup \bar{\alpha}_{j} \cup \alpha_{k} & \\
& \alpha_{i} \cup \delta\left(\alpha_{j}\right), \bar{\alpha}_{i} \cup \delta\left(\alpha_{j}\right), \alpha_{i} \cup \delta\left(\bar{\alpha}_{j}\right), \bar{\alpha}_{i} \cup \delta\left(\bar{\alpha}_{j}\right) &
\end{array}
$$

Proof: This follows from explicit formulas for the cup and the Pontrjagin product, see $[3$, V.3,V.5]. An analogous result is [7, prop 10.3,10.4]. 
We will frequently use the graded commutativity of the cup and Pontrjagin product and identify terms, e.g. when we write $x_{3} \cap \bar{x}_{2} \cap \bar{x}_{1}$ we mean the basis element $-\bar{x}_{1} \cap \bar{x}_{2} \cap x_{3}$.

Note that by construction of the Bockstein homomorphism we have for $v, v^{\prime} \in V$, $v=i_{1} x_{1}+i_{2} x_{2}+i_{3} x_{3}+j_{1} \bar{x}_{1}+j_{2} \bar{x}_{2}+j_{3} \bar{x}_{3}$ and $v^{\prime}=i_{1}^{\prime} x_{1}+i_{2}^{\prime} x_{2}+i_{3}^{\prime} x_{3}+j_{1}^{\prime} \bar{x}_{1}+$ $j_{2}^{\prime} \bar{x}_{2}+j_{3}^{\prime} \bar{x}_{3}$, and for $\{x\}$ the largest integer less than or equal to $x$ :

$$
\delta \alpha_{k}\left(\left[v \mid v^{\prime}\right]\right)=\left\{\frac{i_{k}+i_{k}^{\prime}}{3}\right\}, \quad \delta \bar{\alpha}_{k}\left(\left[v \mid v^{\prime}\right]\right)=\left\{\frac{j_{k}+j_{k}^{\prime}}{3}\right\} .
$$

Proposition 7.2. The following are a description of the $S L\left(\mathbb{F}_{9}\right)$-invariant forms $A, B, C$ and $D$ in terms of our basis of $H^{3}(V, \mathbb{Z} / 3)$ :

$$
\begin{aligned}
A & =\alpha_{1} \alpha_{2} \alpha_{3}-\bar{\alpha}_{1} \bar{\alpha}_{2} \alpha_{3}-\bar{\alpha}_{1} \alpha_{2} \bar{\alpha}_{3}-\alpha_{1} \bar{\alpha}_{2} \bar{\alpha}_{3} \\
B & =\bar{\alpha}_{1} \bar{\alpha}_{2} \bar{\alpha}_{3}-\bar{\alpha}_{1} \alpha_{2} \alpha_{3}-\alpha_{1} \alpha_{2} \bar{\alpha}_{3}-\alpha_{1} \bar{\alpha}_{2} \alpha_{3} \\
C & =\alpha_{3} \delta \alpha_{1}+\alpha_{1} \delta \alpha_{3}-\alpha_{2} \delta \alpha_{2}-\alpha_{1} \delta \alpha_{2}-\alpha_{2} \delta \alpha_{1} \\
& -\bar{\alpha}_{3} \delta \bar{\alpha}_{1}-\bar{\alpha}_{1} \delta \bar{\alpha}_{3}+\bar{\alpha}_{2} \delta \bar{\alpha}_{2}+\bar{\alpha}_{1} \delta \bar{\alpha}_{2}+\bar{\alpha}_{2} \delta \bar{\alpha}_{1} \\
D & =\alpha_{3} \delta \bar{\alpha}_{1}+\alpha_{1} \delta \bar{\alpha}_{3}-\alpha_{2} \delta \bar{\alpha}_{2}-\alpha_{1} \delta \bar{\alpha}_{2}-\alpha_{2} \delta \bar{\alpha}_{1} \\
& +\bar{\alpha}_{3} \delta \alpha_{1}+\bar{\alpha}_{1} \delta \alpha_{3}-\bar{\alpha}_{2} \delta \alpha_{2}-\bar{\alpha}_{1} \delta \alpha_{2}-\bar{\alpha}_{2} \delta \alpha_{1}
\end{aligned}
$$

Proof: Recall that $A, B, C$ and $D$ are expressions of the form

$$
\begin{array}{lllllll}
\Lambda^{3} V & \stackrel{a b c-b a c}{\longrightarrow} & M & \stackrel{t r}{\longrightarrow} & \mathbb{F}_{9} & \rightarrow & \mathbb{F}_{3} \\
S^{2} V & \stackrel{a b}{\longrightarrow} & M & \stackrel{t r}{\longrightarrow} & \mathbb{F}_{9} & \rightarrow & \mathbb{F}_{3} .
\end{array}
$$

Now we just have to calculate the effect of these maps on our basis of $\Lambda^{3} V$ respectively $S^{2} V$ (written additively). For example

$$
\begin{aligned}
A\left(\bar{x}_{1} \cap x_{2} \cap \bar{x}_{3}\right)= & \\
-\chi_{1} \circ \operatorname{tr}\left(\left(\begin{array}{ll}
0 & 0 \\
z & 0
\end{array}\right)\right. & \left.\left(\begin{array}{cc}
1 & -1 \\
0 & -1
\end{array}\right)\left(\begin{array}{cc}
0 & z \\
0 & 0
\end{array}\right)-\left(\begin{array}{cc}
1 & -1 \\
0 & -1
\end{array}\right)\left(\begin{array}{ll}
0 & 0 \\
z & 0
\end{array}\right)\left(\begin{array}{ll}
0 & z \\
0 & 0
\end{array}\right)\right) \\
& =-\chi_{1} \circ \operatorname{tr}\left(\left(\begin{array}{cc}
0 & 0 \\
0 & -1
\end{array}\right)-\left(\begin{array}{ll}
0 & 0 \\
0 & 1
\end{array}\right)\right)=-\chi_{1}(-2)=-1,
\end{aligned}
$$

so we get a contribution $-\bar{\alpha}_{1} \alpha_{2} \bar{\alpha}_{3}$ for $A$.

\section{The Charlap-Vasques description of the differential}

In a situation like ours, Charlap and Vasquez [4] described the differential

$$
d_{2}^{p, q}: E_{2}^{p, q}(\mathbb{Z} / p) \rightarrow E_{2}^{p+2, q-1}(\mathbb{Z} / p)
$$

as follows:

Considering the following cup product

$H^{p}\left(P, H^{q}(V, \mathbb{Z} / 3)\right) \otimes H^{2}\left(P, H^{q-1}(V, \mathbb{Z} / 3) \otimes H_{q}(V, \mathbb{Z} / 3)\right) \stackrel{\cup}{\longrightarrow} H^{p+2}\left(P, H^{q-1}(V, \mathbb{Z} / 3)\right)$, the differential is given by

$$
d_{2}^{p, q}(\xi)=(-1)^{p} \xi \cup\left(V^{q}-Q_{*}(\chi)\right) .
$$

Here $\chi \in H^{2}(P, V)$ is the cohomology class of the extension and $Q_{*}$ the functor $H^{2}(P,-)$ applied to the following map $Q$ induced by Pontrjagin multiplication from the right:

$V=H_{1}(V, \mathbb{Z} / 3) \stackrel{\cap}{\longrightarrow} \operatorname{Hom}_{\mathbb{Z} / 3}\left(H_{q-1}(V, \mathbb{Z} / 3), H_{q}(V, \mathbb{Z} / 3)\right)=H^{q-1}(V, \mathbb{Z} / 3) \otimes H_{q}(V, \mathbb{Z} / 3)$. 
On the other hand $V^{q}$ is universal in the sense that it only depends on the action of $P$ on $V$ and not on the specific extension. We will calculate the term $\xi \cup V^{q}$ in the next section by explicitly calculating the differential in the spectral sequence for the split extension.

In this section we are going to calculate the term $\xi \cup Q_{*}(\chi)$. To do this we have to determine the class $\chi$ of the extension, calculate $Q_{*}$ of $\chi$ and calculate the above cup product.

For the cohomology of $P$ we have the following results:

As $P$ is the direct product of the cyclic groups $T=\langle t\rangle$ and $S=\langle s\rangle$, we will use the tensor product of the minimal resolutions of $T$ and $S$ as our resolution of $P$ : the minimal resolution of $T$ is given by

$$
E .=\ldots \stackrel{N_{t}}{\longrightarrow} \mathbb{Z}[T] \stackrel{t-1}{\longrightarrow} \mathbb{Z}[T] \stackrel{N_{t}}{\longrightarrow} \mathbb{Z}[T] \stackrel{t-1}{\longrightarrow} \mathbb{Z}[T] \rightarrow 0
$$

where $N_{t}=1+t+t^{2}$, and similarly we have the minimal resolution $F$. of $S$. The tensor product of the two resolutions is given by

$$
(E . \otimes F .)_{n}=\bigoplus_{p+q=n} E_{p} \otimes F_{q}, \quad d(e \otimes f)=d e \otimes f+(-1)^{\operatorname{deg} e} e \otimes d f .
$$

Note that $\mathbb{Z}[P]=\mathbb{Z}[T \times S]=\mathbb{Z}[T] \otimes \mathbb{Z}[S]$, so in low degrees the resolution is given by

$$
\ldots \rightarrow \mathbb{Z}[P]^{3} \stackrel{\left(N_{t}, 0\right),(-s+1, t-1),\left(0, N_{s}\right)}{\longrightarrow} \mathbb{Z}[P]^{2} \stackrel{t-1, s-1}{\longrightarrow} \mathbb{Z}[P] \rightarrow 0
$$

The cohomology of $P$ with coefficients in the module $M$ is given by the homology of the complex $Y_{q}=\operatorname{Hom}_{\mathbb{Z}[P]}\left(\mathbb{Z}[P]^{q+1}, M\right)$. We will identify a $\mathbb{Z}[P]$-linear homomorphism $\mathbb{Z}[P]^{q+1}$ with the $q+1$-tupels of images of 1 , ordered in the following way: $E_{q} \otimes F_{0}, E_{q-1} \otimes F_{1}, \ldots$

Lemma 8.1. a) $H^{2}(P, V)=(\mathbb{Z} / 3)^{2}$, a basis for cycles is given by

$$
\left(x_{3}, 0,0\right),\left(\bar{x}_{3}, 0,0\right),\left(0,0, x_{3}\right),\left(0,0, \bar{x}_{3}\right)
$$

and $a$ basis of boundaries is given by $\left(x_{3}, 0,-\bar{x}_{3}\right),\left(\bar{x}_{3}, 0, x_{3}\right)$.

b) $H^{2}\left(P, V^{*}\right)=(\mathbb{Z} / 3)^{2}$, a basis for cycles is given by

$$
\left(\alpha_{1}, 0,0\right),\left(\bar{\alpha}_{1}, 0,0\right),\left(0,0, \alpha_{1}\right),\left(0,0, \bar{\alpha}_{1}\right)
$$

and a basis of boundaries is given by $\left(\alpha_{1}, 0, \bar{\alpha}_{1}\right),\left(\bar{\alpha}_{1}, 0,-\alpha_{1}\right)$.

c) $H^{2}\left(P, \Lambda^{2} V^{*}\right)=(\mathbb{Z} / 3)^{2}$, a basis for cycles is given by

$$
\left(\alpha_{1} \bar{\alpha}_{2}+\bar{\alpha}_{1} \alpha_{2}, 0,0\right),\left(\alpha_{1} \alpha_{2}-\bar{\alpha}_{1} \bar{\alpha}_{2}, 0,0\right),\left(0,0, \alpha_{1} \alpha_{2}-\bar{\alpha}_{1} \bar{\alpha}_{2}\right),\left(0,0, \alpha_{1} \bar{\alpha}_{2}+\bar{\alpha}_{1} \alpha_{2}\right)
$$

and a basis of boundaries is given by

$$
\left(\alpha_{1} \bar{\alpha}_{2}+\bar{\alpha}_{1} \alpha_{2}, 0,-\alpha_{1} \alpha_{2}+\bar{\alpha}_{1} \bar{\alpha}_{2}\right),\left(\alpha_{1} \alpha_{2}-\bar{\alpha}_{1} \bar{\alpha}_{2}, 0, \alpha_{1} \bar{\alpha}_{2}+\bar{\alpha}_{1} \alpha_{2}\right)
$$

Proof: The cycles are given by triples $(a, b, c)$ such that

$$
0=(t-1) a=(s-1) a+N_{t} b=-N_{s} b+(t-1) c=(s-1) c
$$

and the boundaries are given by triples

$$
\left(N_{t} x,(t-1) y-(s-1) x, N_{s} y\right) .
$$

The action of $P$ on $V$ and $V^{*}$ is given by $t$ and $s$ resp. ${ }^{t} t^{-1}$ and ${ }^{t} s^{-1}$, the action of $P$ on $\Lambda^{2} V^{*}$ has to be calculated. We have chosen representants such that the second component is always trivial. 
Since the cocyle of our extension is most easily given in terms of the bar resolution, we need the following comparison between the minimal and bar resolution for cyclic groups, which can easily be proved by induction:

Lemma 8.2. The following is an augmentation preserving chain map from the minimal to the bar resolution of a cyclic group of order $m$ with generator $t$ (necessarily being a homotopy equivalence [3, I 7.5]): In odd degree we take the map

$$
\begin{array}{ccc}
\mathbb{Z}[T] & \rightarrow & \mathbb{Z}[T]\left[T^{2 n+1}\right] \\
1 & \mapsto & \sum\left[t\left|t^{i_{1}}\right| t\left|t^{i_{2}}\right| \ldots\left|t^{i_{n}}\right| t\right]
\end{array}
$$

and in even degree

$$
\begin{array}{ccc}
\mathbb{Z}[T] & \rightarrow & \mathbb{Z}[T]\left[T^{2 n}\right] \\
1 & \mapsto & \sum\left[t^{i_{1}}|t| t^{i_{2}}|\ldots| t^{i_{n}} \mid t\right]
\end{array}
$$

The sum runs over all $n$-tuples $\left(i_{1}, \ldots, i_{n}\right) \in\{0, \ldots, m-1\}^{n}$.

Let $U$ be an extension of $P$ by $V$ and choose a lift $\tilde{a}$ of each element $a$ of $P$ in $U$. Then the cocycle corresponding to the extension is given by

$$
[a \mid b] \mapsto \tilde{a} \tilde{b}(\tilde{a b})^{-1}
$$

Lemma 8.3. A representant of the class $\chi$ of our extension in $\operatorname{Hom}_{\mathbb{Z}[P]}\left(\mathbb{Z}[P]^{3}, V\right)$ is given by $\left(-x_{3}, 0,-\overline{x_{3}}\right)$.

Proof: We have to take the tensor product of the above maps from the minimal to the bar resolution for the groups $T$ and $S$ and calculate the class of the cocycle in the bar resolution. For the first component we get

$$
\begin{array}{ccc}
\mathbb{Z}[P] & \rightarrow & \mathbb{Z}[P][P \times P] \\
1 & \mapsto & \sum_{i=0}^{2}\left[t^{i} \mid t\right]
\end{array}
$$

and for our choice of the lift of $t$ we have

$$
\left[t^{i} \mid t\right] \mapsto\left(\begin{array}{rr}
1 & -i \\
0 & 1
\end{array}\right)\left(\begin{array}{rr}
1 & -1 \\
0 & 1
\end{array}\right)\left(\begin{array}{rr}
1 & i+1 \\
0 & 1
\end{array}\right)=\left\{\begin{array}{ll}
\left(\begin{array}{rr}
1 & -3 \\
0 & 1
\end{array}\right)=-x_{3} & \text { for } i=2 \\
1 & 0 \\
0 & 1
\end{array}\right) \quad \text { otherwise }
$$

Similarly, we get for the second component

$$
1 \mapsto[t \mid s] \mapsto\left(\begin{array}{rr}
1 & -1 \\
0 & 1
\end{array}\right)\left(\begin{array}{rr}
1 & -z \\
0 & 1
\end{array}\right)\left(\begin{array}{rr}
1 & z+1 \\
0 & 1
\end{array}\right)=1
$$

and for the third component

$$
1 \mapsto \sum_{i=0}^{2}\left[s^{i} \mid s\right] \mapsto \sum_{i=0}^{2}\left(\begin{array}{rr}
1 & -i z \\
0 & 1
\end{array}\right)\left(\begin{array}{rr}
1 & -z \\
0 & 1
\end{array}\right)\left(\begin{array}{rr}
1 & (i+1) z \\
0 & 1
\end{array}\right)=-\bar{x}_{3}
$$

The next step is to calculate $Q_{*}(\chi)$ of this element. 
Lemma 8.4. The element $Q_{*}(\chi) \in H^{2}\left(P, H_{3}(V, \mathbb{Z} / 3) \otimes H^{2}(V, \mathbb{Z} / 3)\right)$ is represented by $(u, 0, v)$, where

$$
\begin{aligned}
u= & -\left(x_{1} \cap x_{2} \cap x_{3}\right) \otimes \alpha_{1} \cup \alpha_{2}-\sum_{i \neq 3}\left(x_{i} \cap \bar{x}_{j} \cap x_{3}\right) \otimes \alpha_{i} \cup \bar{\alpha}_{j} \\
& -\sum_{i<j}\left(\bar{x}_{i} \cap \bar{x}_{j} \cap x_{3}\right) \otimes \bar{\alpha}_{i} \cup \bar{\alpha}_{j}-\sum x_{3} \cap \rho\left(x_{i}\right) \otimes \delta \alpha_{i}-\sum x_{3} \cap \rho\left(\bar{x}_{i}\right) \otimes \delta \bar{\alpha}_{i}, \\
v= & -\left(\bar{x}_{1} \cap \bar{x}_{2} \cap \bar{x}_{3}\right) \otimes \bar{\alpha}_{1} \cup \bar{\alpha}_{2}-\sum_{i \neq 3}\left(\bar{x}_{i} \cap x_{j} \cap \bar{x}_{3}\right) \otimes \bar{\alpha}_{i} \cup \alpha_{j} \\
& -\sum_{i<j}\left(x_{i} \cap x_{j} \cap \bar{x}_{3}\right) \otimes \alpha_{i} \cup \alpha_{j}-\sum \bar{x}_{3} \cap \rho\left(x_{i}\right) \otimes \delta \alpha_{i}-\sum \bar{x}_{3} \cap \rho\left(\bar{x}_{i}\right) \otimes \delta \bar{\alpha}_{i} .
\end{aligned}
$$

Proof: To get the components of $Q_{*}(\chi)$, we have to determine what the cup product with $\left(-x_{3}, 0,-\bar{x}_{3}\right)$ does on a basis of $H_{2}(V, \mathbb{Z} / 3)$. For example, $-x_{3}$ sends $x_{i} \cap \bar{x}_{j}$ to $-x_{i} \cap \bar{x}_{j} \cap x_{3}$ and thus gives a contribution $-x_{i} \cap \bar{x}_{j} \cap x_{3} \otimes \alpha_{i} \cup \bar{\alpha}_{j}$ to $u$, or $-\bar{x}_{3}$ sends $\rho x_{i}$ to $-\rho x_{i} \cap \bar{x}_{3}=-\bar{x}_{3} \cap \rho x_{i}$ and thus gives a contribution $-\bar{x}_{3} \cap \rho x_{i} \otimes \delta \alpha_{i}$ to $v$.

Finally we have to calculate the cup product $\xi \cup Q_{*}(\chi)$. For this we have to go into the definition of the cup product:

The cup product of two cocycles $a \in \operatorname{Hom}\left(Y_{i}, M\right)$ and $b \in \operatorname{Hom}\left(Y_{j}, N\right)$ is represented by the map

$$
a \cup b: Y_{i+j} \stackrel{\Delta}{\longrightarrow} Y_{i} \otimes Y_{j} \rightarrow M \otimes N,
$$

where $\Delta$ is a "diagonal approximation", [3, V.3]. For a cyclic group with generator $t$ a diagonal approximation is given in $[3, \mathrm{~V} 1]$ :

$$
\Delta_{i j}(1)= \begin{cases}1 \otimes 1 & i \text { even } \\ 1 \otimes t & i \text { even }, j \text { odd } \\ \sum_{i<j} t^{i} \otimes t^{j} & i, j \text { odd }\end{cases}
$$

We have to work with the tensor product of the approximations for $T$ and $S$ : let $E$. be the resolution for $T$ and $F$. be the resolution for $S$. Then an elements $\xi \in H^{0}\left(P, H^{3}(V, \mathbb{Z} / 3)\right)$ is represented by a map sending $1 \otimes 1 \in E_{0} \otimes F_{0}$ to some cocycle $\omega$ in $H^{3}(V, \mathbb{Z} / 3)$. On the other hand we just calculated that $Q_{*}(\chi)$ is represented by the map sending $(1 \otimes 1,1 \otimes 1,1 \otimes 1) \in\left(E_{2} \otimes F_{0}\right) \oplus\left(E_{1} \otimes F_{1}\right) \oplus\left(E_{0} \otimes F_{2}\right)$ to $(u, 0, v)$ in $H^{2}(V, \mathbb{Z} / 3) \otimes H_{3}(V, \mathbb{Z} / 3)$. Thus a representant of the cup product has the following three components:

$$
\begin{array}{ccclcc}
E_{2} \otimes F_{0} & \stackrel{\Delta \otimes \Delta}{\longrightarrow} & E_{2} \otimes F_{0} \otimes E_{0} \otimes F_{0} & \rightarrow & H^{2}(V, \mathbb{Z} / 3) \otimes H_{3}(V, \mathbb{Z} / 3) \otimes H^{3}(V, \mathbb{Z} / 3) \\
1 \otimes 1 & \mapsto & 1 \otimes 1 \otimes 1 \otimes 1 & \mapsto & u \otimes \omega \\
& & & & \\
E_{1} \otimes F_{1} & \stackrel{\Delta \otimes \Delta}{\longrightarrow} & E_{1} \otimes F_{1} \otimes E_{0} \otimes F_{0} & \rightarrow & H^{2}(V, \mathbb{Z} / 3) \otimes H_{3}(V, \mathbb{Z} / 3) \otimes H^{3}(V, \mathbb{Z} / 3) \\
1 \otimes 1 & \mapsto & 1 \otimes 1 \otimes t \otimes s & \mapsto & 0 \otimes t s \omega \\
& & & & \\
E_{0} \otimes F_{2} & \stackrel{\Delta \otimes \Delta}{\longrightarrow} & E_{0} \otimes F_{2} \otimes E_{0} \otimes F_{0} & \rightarrow & H^{2}(V, \mathbb{Z} / 3) \otimes H_{3}(V, \mathbb{Z} / 3) \otimes H^{3}(V, \mathbb{Z} / 3) \\
1 \otimes 1 & \mapsto & 1 \otimes 1 \otimes 1 \otimes 1 & \mapsto & v \otimes \omega
\end{array}
$$

Evaluating $u \otimes \omega$ and $v \otimes \omega$ we get 
Proposition 8.5. The second term $\xi \cup\left(-Q_{*}(\chi)\right)$ in the Charlap Vasquez description of the differential $d_{2}^{0,3}$ is given by

$$
\begin{array}{ccccc}
A & \mapsto & \left(-\alpha_{1} \cup \alpha_{2}+\bar{\alpha}_{1} \cup \bar{\alpha}_{2}\right. & , 0, & \left.\bar{\alpha}_{1} \cup \alpha_{2}+\alpha_{1} \cup \bar{\alpha}_{2}\right) \\
B & \mapsto & \left(\alpha_{1} \cup \bar{\alpha}_{2}+\bar{\alpha}_{1} \cup \alpha_{2}\right. & , 0, & \left.\alpha_{1} \cup \alpha_{2}-\bar{\alpha}_{1} \cup \bar{\alpha}_{2}\right) \\
C & \mapsto & \left(\delta \alpha_{1}\right. & , 0, & \left.-\delta \bar{\alpha}_{1}\right) \\
D & \mapsto & \left(\delta \bar{\alpha}_{1}\right. & , 0, & \left.\delta \alpha_{1}\right)
\end{array}
$$

\section{The Differential FOR THE SPLit EXTENSION}

Let $\bar{U}$ be the split extension of $P$ by $V$. Let $X_{n}=\mathbb{Z}[V]\left[V^{n}\right]$ be the bar resolution of $\mathrm{V}$. There is an action of $P$ on $X$. by

$$
p * v\left[v_{1}\left|v_{2}\right| \ldots \mid v_{n}\right]=p(v)\left[p\left(v_{1}\right)\left|p\left(v_{2}\right)\right| \ldots \mid p\left(v_{n}\right)\right],
$$

which is compatible with the differential and the augmentation. Let $Y$. be the minimal resolution of $P$, i.e. the tensor product of the minimal resolutions of $T$ and $S$. Then $Y . \otimes X$. is a $\mathbb{Z}[\bar{U}]$-module via the natural action

$$
p(y \otimes x)=p(y) \otimes p(x), \quad v(y \otimes x)=y \otimes v x .
$$

Furthermore $Y . \otimes X$. is a $\mathbb{Z}[\bar{U}]$-free resolution of $\mathbb{Z},[7$, prop 11.1].

Thus we can calculate the cohomology $H^{*}(\bar{U}, \mathbb{Z} / 3)$ as the homology of the double complex

$$
C . .=\operatorname{Hom}_{\mathbb{Z}[\bar{U}]}(Y . \otimes X ., \mathbb{Z} / 3)=\operatorname{Hom}_{\mathbb{Z}[P]}\left(Y ., \operatorname{Hom}_{\mathbb{Z}[V]}(X ., \mathbb{Z} / 3)\right) .
$$

This double complex yields a spectral sequence with

$$
\begin{aligned}
& E_{0}^{p, q}=\operatorname{Hom}_{\mathbb{Z}[P]}\left(Y_{p}, \operatorname{Hom}_{\mathbb{Z}[V]}\left(X_{q}, \mathbb{Z} / 3\right)\right) \\
& E_{2}^{p, q}=H^{p}\left(P, H^{q}(V, \mathbb{Z} / 3)\right)
\end{aligned}
$$

and abutment $H_{\text {total }}^{*}(C)=H^{*}(\bar{U}, \mathbb{Z} / 3)$. One sees as in [7, prop 11.2] that this spectral sequence is the same as the Hochschild-Serre spectral sequence to the extension $1 \rightarrow V \rightarrow \bar{U} \rightarrow P \rightarrow 1$.

The differential for the spectral sequence to the above double complex is calculated as follows see $[9,4.8]$ :

Let $d_{I I}$ be the vertical and $d_{I}$ be the horizontal differential.

$$
\begin{aligned}
E^{0,3} \stackrel{d_{I}=d_{P}}{\longrightarrow} & E^{1,3} \\
d_{I I}=-d_{V} \uparrow & \\
& E^{1,2} \stackrel{d_{I}=d_{P}}{\longrightarrow} E^{2,2}
\end{aligned}
$$

Elements of $Z_{2}^{0,3}$ are of the form $x=x^{0,3}+x^{1,2}$ such that $d_{I I} x^{0,3}=0$ and $d_{I I} x^{1,2}+$ $d_{I} x^{0,3}=0$. They can be identified modulo boundaries with $H^{0}\left(P, H^{3}(V, \mathbb{Z} / 3)\right)$ by projection to $x^{0,3}$. The differential of $x$ is given by

$$
d(x)=\left(d_{I}+d_{I I}\right)\left(x^{0,3}+x^{1,2}\right)=d_{I} x^{0,3}+d_{I I} x^{0,3}+d_{I} x^{1,2}+d_{I I} x^{1,2}=d_{I} x^{1,2} .
$$

In our case $\alpha \in\{A, B, C, D\}$ we have $d_{I I} \alpha=0$ and we have to find an element $\beta \in E_{0}^{1,2}$ such that $d_{I I} \beta+d_{I} \alpha=0$. Then we have to calculate $d_{I} \beta$ and the resulting element of $E_{0}^{2,2}$ will represent an element of $H^{2}\left(P, H^{2}(V, \mathbb{Z} / 3)\right)$.

As we have

$$
E_{0}^{p, q}=\operatorname{Hom}_{\mathbb{Z}[P]}\left(\mathbb{Z}[P]^{p+1}, \operatorname{Hom}_{\mathbb{Z}[V]}\left(X_{q}, \mathbb{Z} / 3\right)\right),
$$


we will identify a $\mathbb{Z}[P]$-linear homomorphism $\mathbb{Z}[P]^{p+1} \rightarrow \operatorname{Hom}_{\mathbb{Z}[P]}\left(X_{q}, \mathbb{Z} / 3\right)$ with the $p+1$-tupel of images of 1 . Similarly we have $\operatorname{Hom}_{\mathbb{Z}[V]}\left(X_{q}, \mathbb{Z} / 3\right)=\operatorname{Hom}_{\mathbb{Z}[V]}\left(\mathbb{Z}[V]\left[V^{q}\right], \mathbb{Z} / 3\right)$ and we will identify an element of this group with a map $V^{q} \rightarrow \mathbb{Z} / 3$.

So for a representant of $\alpha$ we have to calculate $d_{I} \alpha=d_{P} \alpha$. With the above identifications this element has components $d_{P}(\alpha)_{1}=(t-1) \alpha$, and $d_{P}(\alpha)_{2}=$ $(s-1) \alpha$.

Then we have to find an element $\beta$ of $E_{0}^{1,2}$ such that $-d_{I I} \beta=d_{V} \beta=d_{I} \alpha$. The differential $d_{V}$ is given by

$$
d_{V}(f)[a|b| c]=f[a \mid b]-f[a+b \mid c]+f[a \mid b+c]-f[b \mid c]
$$

on each component.

The next step is to calculate the differential $d_{I}=d_{P}$ of $\beta=\left(\beta_{1}, \beta_{2}\right)$ : With the above identifications it has the three components $N_{t}\left(\beta_{1}\right),-(s-1) \beta_{1}+(t-1) \beta_{2}$ and $N_{s}\left(\beta_{2}\right)$ respectively.

Finally we will show that that some of the resulting cocycles become zero in $E_{2}^{2,2}=H^{2}\left(P, H^{2}(V, \mathbb{Z} / 3)\right)$ by exhibiting them as boundaries from $E_{0}^{2,1}$.

We will proceed for $A, B \in\left(\Lambda^{3} V^{*}\right)^{P}$ and $C, D \in\left(S^{2} V^{*}\right)^{P}$ separately. We will only give the results of the calculation and indicate how the calculations can be done. All verifications are left to the reader.

The following will be the result of the appendix:

Proposition 9.1. Let $A, B, C$ and $D$ as in proposition 7.2. Then the three components for the differential in $E_{2}^{2,2}(\mathbb{Z} / 3)$ are:

$$
\begin{array}{cccc}
A: & \left(-\alpha_{1} \alpha_{2}+\bar{\alpha}_{1} \bar{\alpha}_{2}-\delta \alpha_{1}\right. & , 0, & \left.\bar{\alpha}_{1} \alpha_{2}+\alpha_{1} \bar{\alpha}_{2}+\delta \bar{\alpha}_{1}\right) \\
B: & \left(\alpha_{1} \bar{\alpha}_{2}+\bar{\alpha}_{1} \alpha_{2}-\delta \bar{\alpha}_{1}\right. & , 0, & \left.\alpha_{1} \alpha_{2}-\bar{\alpha}_{1} \bar{\alpha}_{2}-\delta \alpha_{1}\right) \\
C: & \left(\delta \alpha_{1}+\alpha_{1} \alpha_{2}-\bar{\alpha}_{1} \bar{\alpha}_{2}\right. & , 0, & \left.-\delta \bar{\alpha}_{1}-\alpha_{1} \bar{\alpha}_{2}-\bar{\alpha}_{1} \alpha_{2}\right) \\
D: & \left(\delta \bar{\alpha}_{1}+\alpha_{1} \bar{\alpha}_{2}+\bar{\alpha}_{1} \alpha_{2}\right. & , 0, & \left.\delta \alpha_{1}+\alpha_{1} \alpha_{2}-\bar{\alpha}_{1} \bar{\alpha}_{2}\right)
\end{array}
$$

b) The three components of the differential in $E_{2}^{2,2}(\mathbb{Z})$ are given by

$$
\begin{array}{cccc}
A: & \left(-\alpha_{1} \alpha_{2}+\bar{\alpha}_{1} \bar{\alpha}_{2}\right. & , 0, & \left.\bar{\alpha}_{1} \alpha_{2}+\alpha_{1} \bar{\alpha}_{2}\right) \\
B: & \left(\alpha_{1} \bar{\alpha}_{2}+\bar{\alpha}_{1} \alpha_{2}\right. & , 0, & \left.\alpha_{1} \alpha_{2}-\bar{\alpha}_{1} \bar{\alpha}_{2}\right) \\
C: & \left(\alpha_{1} \alpha_{2}-\bar{\alpha}_{1} \bar{\alpha}_{2}\right. & , 0, & \left.-\alpha_{1} \bar{\alpha}_{2}-\bar{\alpha}_{1} \alpha_{2}\right) \\
D: & \left(\alpha_{1} \bar{\alpha}_{2}+\bar{\alpha}_{1} \alpha_{2}\right. & , 0, & \left.\alpha_{1} \alpha_{2}-\bar{\alpha}_{1} \bar{\alpha}_{2}\right)
\end{array}
$$

Proof: a) 8.5, A.5, B.4

b) Obvious from a).

\section{Appendix A. The Differential For $\Lambda^{3} V^{*}$}

Let $a_{i}$ be $\alpha_{i}$ considered as a map $V \rightarrow \mathbb{Z} / 3$. Then $a_{i} a_{j} a_{k}: V^{3} \rightarrow \mathbb{Z} / 3$ represents $\alpha_{i} \alpha_{j} \alpha_{k}$ etc..

Lemma A.1. The image of $A$ and $B$ in $E_{0}^{1,3}$ are given by

$$
\begin{array}{r}
A_{1}^{1,3}=a_{1} a_{1} a_{2}+a_{1} a_{1} a_{3}+a_{1} a_{2} a_{2}-a_{1} \bar{a}_{1} \bar{a}_{2}-a_{1} \bar{a}_{1} \bar{a}_{3}-a_{1} \bar{a}_{2} \bar{a}_{2}-\bar{a}_{1} a_{1} \bar{a}_{2}-\bar{a}_{1} a_{1} \bar{a}_{3} \\
-\bar{a}_{1} a_{2} \bar{a}_{2}-\bar{a}_{1} \bar{a}_{1} a_{2}-\bar{a}_{1} \bar{a}_{1} a_{3}-\bar{a}_{1} \bar{a}_{2} a_{2}
\end{array}
$$




$$
\begin{aligned}
& A_{2}^{1,3}=-a_{1} a_{1} a_{1}-a_{1} a_{1} a_{2}-a_{1} a_{1} \bar{a}_{1}-a_{1} a_{1} \bar{a}_{3}+a_{1} a_{2} a_{1}-a_{1} a_{2} \bar{a}_{1}-a_{1} a_{2} \bar{a}_{2}-a_{1} \bar{a}_{1} a_{1} \\
& -a_{1} \bar{a}_{1} a_{3}+a_{1} \bar{a}_{1} \bar{a}_{1}+a_{1} \bar{a}_{1} \bar{a}_{2}-a_{1} \bar{a}_{2} a_{1}-a_{1} \bar{a}_{2} a_{2}-a_{1} \bar{a}_{2} \bar{a}_{1}-\bar{a}_{1} a_{1} a_{1}-\bar{a}_{1} a_{1} a_{3} \\
& +\bar{a}_{1} a_{1} \bar{a}_{1}+\bar{a}_{1} a_{1} \bar{a}_{2}-\bar{a}_{1} a_{2} a_{1}-\bar{a}_{1} a_{2} a_{2}-\bar{a}_{1} a_{2} \bar{a}_{1}+\bar{a}_{1} \bar{a}_{1} a_{1}+\bar{a}_{1} \bar{a}_{1} a_{2}+\bar{a}_{1} \bar{a}_{1} \bar{a}_{1} \\
& +\bar{a}_{1} \bar{a}_{1} \bar{a}_{3}-\bar{a}_{1} \bar{a}_{2} a_{1}+\bar{a}_{1} \bar{a}_{2} \bar{a}_{1}+\bar{a}_{1} \bar{a}_{2} \bar{a}_{2} \\
& B_{1}^{1,3}=-a_{1} a_{1} \bar{a}_{2}-a_{1} a_{1} \bar{a}_{3}-a_{1} a_{2} \bar{a}_{2}-a_{1} \bar{a}_{1} a_{2}-a_{1} \bar{a}_{1} a_{3}-a_{1} \bar{a}_{2} a_{2}-\bar{a}_{1} a_{1} a_{2}-\bar{a}_{1} a_{1} a_{3} \\
& -\bar{a}_{1} a_{2} a_{2}+\bar{a}_{1} \bar{a}_{1} \bar{a}_{2}+\bar{a}_{1} \bar{a}_{1} \bar{a}_{3}+\bar{a}_{1} \bar{a}_{2} \bar{a}_{2} \\
& B_{1}^{1,3}=-a_{1} a_{1} a_{1}-a_{1} a_{1} a_{3}+a_{1} a_{1} \bar{a}_{1}+a_{1} a_{1} \bar{a}_{2}-a_{1} a_{2} a_{1}-a_{1} a_{2} a_{2}-a_{1} a_{2} \bar{a}_{1}+a_{1} \bar{a}_{1} a_{1} \\
& +a_{1} \bar{a}_{1} a_{2}+a_{1} \bar{a}_{1} \bar{a}_{1}+a_{1} \bar{a}_{1} \bar{a}_{3}-a_{1} \bar{a}_{2} a_{1}+a_{1} \bar{a}_{2} \bar{a}_{1}+a_{1} \bar{a}_{2} \bar{a}_{2}+\bar{a}_{1} a_{1} a_{1}+\bar{a}_{1} a_{1} a_{2} \\
& +\bar{a}_{1} a_{1} \bar{a}_{1}+\bar{a}_{1} a_{1} \bar{a}_{3}-\bar{a}_{1} a_{2} a_{1}+\bar{a}_{1} a_{2} \bar{a}_{1}+\bar{a}_{1} a_{2} \bar{a}_{2}+\bar{a}_{1} \bar{a}_{1} a_{1}+\bar{a}_{1} \bar{a}_{1} a_{3}-\bar{a}_{1} \bar{a}_{1} \bar{a}_{1} \\
& -\bar{a}_{1} \bar{a}_{1} \bar{a}_{2}+\bar{a}_{1} \bar{a}_{2} a_{1}+\bar{a}_{1} \bar{a}_{2} a_{2}+\bar{a}_{1} \bar{a}_{2} \bar{a}_{1} \text {. }
\end{aligned}
$$

Proof: One has to calculate $t-1$ and $s-1$ of $A$ and $B$.

Lemma A.2. Define the following maps $V \rightarrow \mathbb{Z} / 3$ for $v=i_{1} x_{1}+i_{2} x_{2}+i_{3} x_{3}+$ $j_{1} \bar{x}_{1}+j_{2} \bar{x}_{2}+j_{3} \bar{x}_{3}$

$$
\begin{aligned}
u_{n}(v) & =i_{n}^{2}, & \bar{u}_{n}(v) & =j_{n}^{2} \\
w_{n, m}(v) & =-i_{n} i_{m}, & \bar{w}_{n, m}(v) & =-j_{n} j_{m} \\
v_{n, m}(v) & =-i_{n} j_{m} & &
\end{aligned}
$$

Then the following are lifts of $A$ and $B$ to $E_{0}^{1,2}$, i.e. $d_{V} A^{1,2}=d_{P} A^{0,3}$ and $d_{V} B^{1,2}=$ $d_{P} B^{0,3}$ :

$$
\begin{gathered}
A_{1}^{1,2}=a_{1} \bar{u}_{2}-a_{1} u_{2}+\bar{a}_{1} v_{2,2}-\bar{u}_{1} a_{2}-\bar{u}_{1} a_{3}+u_{1} a_{2}+u_{1} a_{3}-v_{1,1} \bar{a}_{2}-v_{1,1} \bar{a}_{3} \\
A_{2}^{1,2}=-a_{1} \bar{u}_{1}+a_{1} v_{2,1}+a_{1} v_{2,2}+a_{2} u_{1}-\bar{a}_{1} \bar{u}_{2}+\bar{a}_{1} u_{2}-\bar{a}_{1} v_{1,1}+\bar{a}_{1} v_{2,1}+\bar{a}_{1} w_{12}+\bar{a}_{2} \bar{u}_{1} \\
-\bar{a}_{2} u_{1}-\bar{a}_{2} v_{1,1}-\bar{u}_{1} a_{2}+\bar{u}_{1} \bar{a}_{1}+\bar{u}_{1} \bar{a}_{3}-\bar{w}_{1,2} a_{1}+\bar{w}_{1,2} \bar{a}_{1}-u_{1} a_{1}-u_{1} a_{2}-u_{1} \bar{a}_{1} \\
\quad-u_{1} \bar{a}_{3}-v_{1,1} a_{1}+v_{1,1} a_{2}-v_{1,1} a_{3}+v_{1,1} \bar{a}_{2}-v_{1,2} a_{1}-v_{1,2} \bar{a}_{1}+w_{1,2} a_{1} \\
B_{1}^{1,2}=a_{1} v_{2,2}-\bar{a}_{1} \bar{u}_{2}+\bar{a}_{1} u_{2}+\bar{u}_{1} \bar{a}_{2}+\bar{u}_{1} \bar{a}_{3}-u_{1} \bar{a}_{2}-u_{1} \bar{a}_{3}-v_{1,1} a_{2}-v_{1,1} a_{3} \\
B_{2}^{1,2}=-a_{1} \bar{u}_{1}-a_{1} \bar{u}_{2}+a_{1} u_{2}+a_{1} v_{2,1}-a_{2} u_{1}-\bar{a}_{1} v_{1,1}-\bar{a}_{1} v_{2,1}-\bar{a}_{1} v_{2,2}+\bar{a}_{1} w_{1,2}+\bar{a}_{2} \bar{u}_{1} \\
-\bar{a}_{2} u_{1}+\bar{a}_{2} v_{1,1}-\bar{u}_{1} a_{2}+\bar{u}_{1} a_{3}-\bar{u}_{1} \bar{a}_{1}-\bar{u}_{1} \bar{a}_{2}+\bar{w}_{1,2} a_{1}+\bar{w}_{1,2} \bar{a}_{1}-u_{1} a_{1}-u_{1} a_{3} \\
+u_{1} \bar{a}_{1}+u_{1} \bar{a}_{2}+v_{1,1} a_{1}-v_{1,1} a_{2}+v_{1,1} \bar{a}_{3}-v_{1,2} a_{1}+v_{1,2} \bar{a}_{1}-w_{1,2} a_{1}
\end{gathered}
$$

Proof: First one has to verify the following equations of functions $V^{2} \rightarrow \mathbb{Z} / 3$ :

$$
\begin{aligned}
a_{n} a_{n} & =d_{V} u_{n} \\
\bar{a}_{n} \bar{a}_{n} & =d_{V} \bar{u}_{n} \\
a_{n} \bar{a}_{m}+a_{m} \bar{a}_{n} & =d_{V} v_{n, m} \\
a_{n} a_{m}+a_{m} a_{n} & =d_{V} w_{n, m} \\
\bar{a}_{n} \bar{a}_{m}+\bar{a}_{m} \bar{a}_{n} & =d_{V} \bar{w}_{n, m} .
\end{aligned}
$$

Then one uses these equations and $d_{V}\left(a_{n}\right)=d_{V}\left(\bar{a}_{n}\right)=0$ to write the expressions of the last lemma as images of $-d_{V}$, for example

$$
a_{1} a_{1} a_{2}=d_{V}\left(u_{1}\right) a_{2}=d_{V}\left(u_{1} a_{2}\right) .
$$


Lemma A.3. The three components of $d_{P} A^{1,2}$ and $d_{P} B^{1,2}$ in $E_{0}^{2,2}$ are given as follows:

$$
\begin{aligned}
& A_{1}^{2,2}=-a_{1} \bar{u}_{1}+a_{1} u_{1}-\bar{a}_{1} v_{1,1}-\bar{u}_{1} a_{1}+u_{1} a_{1}-v_{1,1} \bar{a}_{1} \\
& A_{2}^{2,2}=a_{1} \bar{u}_{1}-a_{1} v_{1,1}-\bar{a}_{1} u_{1}+\bar{a}_{1} v_{1,1}+\bar{u}_{1} a_{1}-u_{1} \bar{a}_{1}-v_{1,1} a_{1}+v_{1,1} \bar{a}_{1} \\
& A_{3}^{2,2}=a_{1} v_{1,1}-\bar{a}_{1} \bar{u}_{1}+\bar{a}_{1} u_{1}-\bar{u}_{1} \bar{a}_{1}+u_{1} \bar{a}_{1}+v_{1,1} a_{1} \\
& B_{1}^{2,2}=-a_{1} v_{1,1}+\bar{a}_{1} \bar{u}_{1}-\bar{a}_{1} u_{1}+\bar{u}_{1} \bar{a}_{1}-u_{1} \bar{a}_{1}-v_{1,1} a_{1} \\
& B_{2}^{2,2}=-a_{1} \bar{u}_{1}-a_{1} v_{1,1}-\bar{a}_{1} u_{1}-\bar{a}_{1} v_{1,1}-\bar{u}_{1} a_{1}-u_{1} \bar{a}_{1}-v_{1,1} a_{1}-v_{1,1} \bar{a}_{1} \\
& B_{3}^{2,2}=-a_{1} \bar{u}_{1}+a_{1} u_{1}-\bar{a}_{1} v_{1,1}-\bar{u}_{1} a_{1}+u_{1} a_{1}-v_{1,1} \bar{a}_{1}
\end{aligned}
$$

Proof: We have to calculate $N_{t} A_{1}^{1,2},-(s-1) A_{1}^{1,2}+(t-1) A_{2}^{1,2}$ and $N_{s} A_{2}^{1,2}$ and similarly for $B$. The action of $t$ and $s$ on $a_{1}, \bar{a}_{1}, u_{1}, \bar{u}_{1}$ and $v_{1,1}$ is trivial and on the other terms given as follows:

$$
\begin{array}{rlrl}
t a_{2} & =a_{2}+a_{1} & s a_{2} & =a_{2}+2 \bar{a}_{1} \\
t \bar{a}_{2} & =\bar{a}_{2}+\bar{a}_{1} & s \bar{a}_{2} & =\bar{a}_{2}+a_{1} \\
t a_{3} & =a_{3}+a_{2} & s a_{3} & =a_{3}+a_{1}+2 \bar{a}_{1}+2 \bar{a}_{2} \\
t \bar{a}_{3} & =\bar{a}_{3}+\bar{a}_{2} & s \bar{a}_{3} & =\bar{a}_{3}+\bar{a}_{1}+a_{1}+a_{2} \\
t u_{2} & =u_{2}+u_{1}+w_{1,2} & s u_{2} & =u_{2}+\bar{u}_{1}-v_{2,1} \\
t \bar{u}_{2} & =\bar{u}_{2}+\bar{u}_{1}+\bar{w}_{1,2} & s \bar{u}_{2} & =\bar{u}_{2}+u_{1}+v_{1,2} \\
t w_{1,2} & =w_{1,2}+2 u_{1} & s w_{1,2} & =w_{1,2}+2 v_{1,1} \\
t \bar{w}_{1,2} & =\bar{w}_{1,2}+2 \bar{u}_{1} & s \bar{w}_{1,2} & =\bar{w}_{1,2}+v_{1,1} \\
t v_{1,2} & =v_{1,2}+v_{1,1} & s v_{1,2} & =v_{1,2}+2 u_{1} \\
t v_{2,1} & =v_{2,1}+v_{1,1} & s v_{2,1} & =v_{2,1}+\bar{u}_{1} \\
t v_{2,2} & =v_{2,2}+v_{1,1}+v_{1,2}+v_{2,1} & s v_{2,2} & =v_{2,2}+w_{1,2}+2 \bar{w}_{1,2}+2 v_{1,1}
\end{array}
$$

For example

$$
s \bar{w}_{1,2}(v)=\bar{w}_{1,2}\left(s^{-1} v\right)=-j_{1}\left(j_{2}+i_{1}\right)=\bar{w}_{1,2}(v)+v_{1,1}(v) .
$$

Lemma A.4. Let $c h_{n, m}$ be the characteristic function which is 1 on $n x_{1}+m \bar{x}_{1}$ and 0 on all other elements of $V$. Let $d_{V}: E_{0}^{2,1} \rightarrow E_{0}^{2,2}$ be the boundary given by $d_{V}(f)[v \mid w]=f[w]-f[v+w]+f[v]$ on each component. Then we have the following equations in $E_{2}^{2,2}$ :

$$
\begin{aligned}
& A_{1}^{2,2}=-\delta a_{1}+d_{V}\left(c h_{1,1}+c h_{1,2}+c h_{2,0}\right) \\
& A_{2}^{2,2}=d_{V}\left(c h_{1,2}-c h_{2,1}\right) \\
& A_{3}^{2,2}=\delta \bar{a}_{1}-d_{V}\left(c h_{0,2}+c h_{1,1}+c h_{2,1}\right) \\
& B_{1}^{2,2}=-\delta \bar{a}_{1}+d_{V}\left(c h_{0,2}+c h_{1,1}+c h_{2,1}\right) \\
& B_{2}^{2,2}=d_{V}\left(c h_{2,2}-c h_{1,1}\right) \\
& B_{3}^{2,2}=-\delta a_{1}+d_{V}\left(c h_{1,1}+c h_{1,2}+c h_{2,0}\right)
\end{aligned}
$$

Proof: This has to be proved by inspection. Since only the coefficients of $x_{1}$ and $\bar{x}_{1}$ of elements in $V$ are involved, one has to check that the above functions agree 
on all 81 elements of $\left\langle x_{1}, \bar{x}_{1}\right\rangle^{2} \subseteq V^{2}$.

Finally the lemmas prove the following proposition:

Proposition A.5. The first term $\xi \cup V^{3}$ in the Charlap Vasquez description of the differential $d_{2}^{0,3}$ is given by

$$
\begin{aligned}
& A \mapsto\left(-\delta \alpha_{1}, 0, \delta \bar{\alpha}_{1}\right) \\
& B \mapsto\left(-\delta \bar{\alpha}_{1}, 0,-\delta \alpha_{1}\right)
\end{aligned}
$$

\section{Appendix B. The Differential For $S^{2} V^{*}$}

Lemma B.1. Let $\{x\}$ be the largest integer less than or equal to $x$ and define the following functions on $v \in V, v=i_{1} x_{1}+i_{2} x_{2}+i_{3} x_{3}+j_{1} \bar{x}_{1}+j_{2} \bar{x}_{2}+j_{3} \bar{x}_{3}$ :

$$
\begin{array}{ll}
p_{2}=-\left\{\frac{i_{1}+i_{2}}{3}\right\} & q_{2}=-\left\{\frac{i_{2}+2 j_{1}}{3}\right\} \\
\bar{p}_{2}=-\left\{\frac{j_{1}+j_{2}}{3}\right\} & \bar{q}_{2}=-\left\{\frac{j_{2}+i_{1}}{3}\right\} \\
p_{3}=-\left\{\frac{i_{3}+i_{2}}{3}\right\} & q_{3}=-\left\{\frac{i_{3}+2 j_{2}+2 j_{1}+i_{1}}{3}\right\} \\
\bar{p}_{3}=-\left\{\frac{j_{3}+j_{2}}{3}\right\} & \bar{q}_{3}=-\left\{\frac{j_{3}+i_{2}+j_{1}+i_{1}}{3}\right\}
\end{array}
$$

Then the action of $t$ and $s$ on terms of the form $\delta a_{i}$ and $\delta \bar{a}_{i}$ can be described as follows:

$$
\begin{array}{rlrl}
t \delta a_{1} & =\delta a_{1} & s \delta a_{1}=\delta a_{1} \\
t \delta \bar{a}_{1}=\delta \bar{a}_{1} & s \delta \bar{a}_{1}=\delta \bar{a}_{1} \\
t \delta a_{2}=\delta a_{2}+\delta a_{1}+d_{V} p_{2} & s \delta a_{2}=\delta a_{2}-\delta \bar{a}_{1}+d_{V} q_{2} \\
t \delta \bar{a}_{2}=\delta \bar{a}_{2}+\delta \bar{a}_{1}+d_{V} \bar{p}_{2} & s \delta \bar{a}_{2}=\delta \bar{a}_{2}+\delta a_{1}+d_{V} \bar{q}_{2} \\
t \delta a_{3}=\delta a_{3}+\delta a_{2}+d_{V} p_{3} & s \delta a_{3}=\delta a_{3}-\delta \bar{a}_{2}-\delta \bar{a}_{1}+\delta a_{1}+d_{V} q_{3} \\
t \delta \bar{a}_{3}=\delta \bar{a}_{3}+\delta \bar{a}_{2}+d_{V} \bar{p}_{3} & s \delta \bar{a}_{3}=\delta \bar{a}_{3}+\delta a_{2}+\delta a_{1}+\delta \bar{a}_{1}+d_{V} \bar{q}_{3}
\end{array}
$$

Proof: As in $[7,11.9]$ : define $\langle a\rangle \in\{0,1,2\}$ to be the principal representative of $a$ mod 3 and let $v, v^{\prime}$ be two elements of $V, v=i_{1} x_{1}+i_{2} x_{2}+i_{3} x_{3}+j_{1} \bar{x}_{1}+j_{2} \bar{x}_{2}+j_{3} \bar{x}_{3}$, $v^{\prime}=i_{1}^{\prime} x_{1}+i_{2}^{\prime} x_{2}+i_{3}^{\prime} x_{3}+j_{1}^{\prime} \bar{x}_{1}+j_{2}^{\prime} \bar{x}_{2}+j_{3}^{\prime} \bar{x}_{3}$. We will calculate the example $s \delta a_{3}$. We have

$$
\begin{array}{r}
i_{3}+i_{3}^{\prime}+2 j_{2}+2 j_{2}^{\prime}+2 j_{1}+2 j_{1}^{\prime}+i_{1}+i_{1}^{\prime}=3\left\{\frac{i_{3}+2 j_{2}+2 j_{1}+i_{1}}{3}\right\}+3\left\{\frac{i_{3}^{\prime}+2 j_{2}^{\prime}+2 j_{1}^{\prime}+i_{1}^{\prime}}{3}\right\}+ \\
\left\langle i_{3}+2 j_{2}+2 j_{1}+i_{1}\right\rangle+\left\langle i_{3}^{\prime}+2 j_{2}^{\prime}+2 j_{1}^{\prime}+i_{1}^{\prime}\right\rangle
\end{array}
$$

and thus

$$
\begin{aligned}
&\left\{\frac{i_{3}+i_{3}^{\prime}+2 j_{2}+2 j_{2}^{\prime}+2 j_{1}+2 j_{1}^{\prime}+i_{1}+i_{1}^{\prime}}{3}\right\}=\left\{\frac{i_{3}+2 j_{2}+2 j_{1}+i_{1}}{3}\right\}+\left\{\frac{i_{3}^{\prime}+2 j_{2}^{\prime}+2 j_{1}^{\prime}+i_{1}^{\prime}}{3}\right\}+ \\
&\left\{\frac{\left.\left\langle i_{3}+2 j_{2}+2 j_{1}+i_{1}\right\rangle+\left\langle i_{3}^{\prime}+2 j_{2}^{\prime}+2 j_{1}^{\prime}+i_{1}^{\prime}\right)\right\rangle}{3}\right\} .
\end{aligned}
$$

Similarly

$$
\begin{aligned}
\left\{\frac{i_{3}+i_{3}^{\prime}+2 j_{2}+2 j_{2}^{\prime}+2 j_{1}+2 j_{1}^{\prime}+i_{1}+i_{1}^{\prime}}{3}\right\}=\left\{\frac{i_{3}+i_{3}^{\prime}}{3}\right\}+ & 2\left\{\frac{j_{2}+j_{2}^{\prime}}{3}\right\}+2\left\{\frac{j_{1}+j_{1}^{\prime}}{3}\right\}+\left\{\frac{i_{1}+i_{1}^{\prime}}{3}\right\}+ \\
& \left\{\frac{\left\langle i_{3}+i_{3}^{\prime}\right\rangle+2\left\langle j_{2}+j_{2}^{\prime}\right\rangle+2\left\langle j_{1}+j_{1}^{\prime}\right\rangle+\left\langle i_{1}+i_{1}^{\prime}\right\rangle}{3}\right\} .
\end{aligned}
$$

Finally, using these equations and recalling the definition of $d_{V}$, we get

$$
\begin{aligned}
& s \delta a_{3}\left(v, v^{\prime}\right)=\delta a_{3}\left(s^{-1} v, s^{-1} v^{\prime}\right)=\left\{\frac{\left\langle i_{3}+2 j_{2}+2 j_{1}+i_{1}\right\rangle+\left\langle i_{3}^{\prime}+2 j_{2}^{\prime}+2 j_{1}^{\prime}+i_{1}^{\prime}\right\rangle}{3}\right\} \\
& =\left\{\frac{i_{3}+i_{3}^{\prime}}{3}\right\}+2\left\{\frac{j_{2}+j_{2}^{\prime}}{3}\right\}+2\left\{\frac{j_{1}+j_{1}^{\prime}}{3}\right\}+\left\{\frac{i_{1}+i_{1}^{\prime}}{3}\right\}-\left\{\frac{i_{3}+2 j_{2}+2 j_{1}+i_{1}}{3}\right\}-\left\{\frac{i_{3}^{\prime}+2 j_{2}^{\prime}+2 j_{1}^{\prime}+i_{1}^{\prime}}{3}\right\} \\
& +\left\{\frac{\left\langle i_{3}+i_{3}^{\prime}\right\rangle+2\left\langle j_{2}+j_{2}^{\prime}\right\rangle+2\left\langle j_{1}+j_{1}^{\prime}\right\rangle+\left\langle i_{1}+i_{1}^{\prime}\right\rangle}{3}\right\} \\
& =\delta a_{3}\left(v, v^{\prime}\right)+2 \delta \bar{a}_{2}\left(v, v^{\prime}\right)+2 \delta \bar{a}_{1}\left(v, v^{\prime}\right)+\delta a_{1}\left(v, v^{\prime}\right)+d_{V} q_{3}\left(v, v^{\prime}\right) .
\end{aligned}
$$


Lemma B.2. a) The image of $C$ and $D$ in $E_{0}^{1,3}$ are given by

$C_{1}^{1,3}=a_{1} d_{V} p_{2}+a_{1} d_{V} p_{3}-a_{2} d_{V} p_{2}-\bar{a}_{1} d_{V} \bar{p}_{2}-\bar{a}_{1} d_{V} \bar{p}_{3}+\bar{a}_{2} d_{V} \bar{p}_{2}$

$C_{2}^{1,3}=-a_{1} d_{V} q_{2}+a_{1} d_{V} q_{3}+a_{1} d_{V} \bar{q}_{2}-a_{2} d_{V} q_{2}+\bar{a}_{1} d_{V} q_{2}+\bar{a}_{1} d_{V} \bar{q}_{2}-\bar{a}_{1} d_{V} \bar{q}_{3}+\bar{a}_{2} d_{V} \bar{q}_{2}$

$D_{1}^{1,3}=a_{1} d_{V} \bar{p}_{2}+a_{1} d_{V} \bar{p}_{3}-a_{2} d_{V} \bar{p}_{2}+\bar{a}_{1} d_{V} p_{2}+\bar{a}_{1} d_{V} p_{3}-\bar{a}_{2} d_{V} p_{2}$

$D_{2}^{1,3}=-a_{1} d_{V} q_{2}-a_{1} d_{V} \bar{q}_{2}+a_{1} d_{V} \bar{q}_{3}-a_{2} d_{V} \bar{q}_{2}-\bar{a}_{1} d_{V} q_{2}+\bar{a}_{1} d_{V} q_{3}+\bar{a}_{1} d_{V} \bar{q}_{2}-\bar{a}_{2} d_{V} q_{2}$

b) The lifts of $C$ and $D$ to $E_{0}^{1,2}$ are given as follows:

$$
\begin{aligned}
& C_{1}^{1,2}=-\left(a_{1} p_{2}+a_{1} p_{3}-a_{2} p_{2}-\bar{a}_{1} \bar{p}_{2}-\bar{a}_{1} \bar{p}_{3}+\bar{a}_{2} \bar{p}_{2}\right) \\
& C_{2}^{1,2}=-\left(-a_{1} q_{2}+a_{1} q_{3}+a_{1} \bar{q}_{2}-a_{2} q_{2}+\bar{a}_{1} q_{2}+\bar{a}_{1} \bar{q}_{2}-\bar{a}_{1} \bar{q}_{3}+\bar{a}_{2} \bar{q}_{2}\right) \\
& D_{1}^{1,2}=-\left(a_{1} \bar{p}_{2}+a_{1} \bar{p}_{3}-a_{2} \bar{p}_{2}+\bar{a}_{1} p_{2}+\bar{a}_{1} p_{3}-\bar{a}_{2} p_{2}\right) \\
& D_{2}^{1,2}=-\left(-a_{1} q_{2}-a_{1} \bar{q}_{2}+a_{1} \bar{q}_{3}-a_{2} \bar{q}_{2}-\bar{a}_{1} q_{2}+\bar{a}_{1} q_{3}+\bar{a}_{1} \bar{q}_{2}-\bar{a}_{2} q_{2}\right)
\end{aligned}
$$

Proof: a) We have to calculate $t-1$ and $s-1$ of the terms of $C$ and $D$, respectively This is easily done with the last lemma.

b) Use the fact that $d_{V} a_{i}=d_{V} \bar{a}_{i}=0$ and that $d_{V}$ is a derivation, e.g $d_{V}\left(a_{1} p_{2}\right)=$ $d_{V}\left(a_{1}\right) p_{2}-a_{1} d_{V}\left(p_{2}\right)=-a_{1} d_{V}\left(p_{2}\right)$.

Lemma B.3. Let $x$ be any function on $V$, then we have

a)

$$
\begin{array}{lll}
t\left(a_{1} x\right)=a_{1} t x & t\left(\bar{a}_{1} x\right)=\bar{a}_{1} t x \\
s\left(a_{1} x\right)=a_{1} s x & s\left(\bar{a}_{1} x\right)=\bar{a}_{1} s x
\end{array}
$$

b)

$$
\begin{array}{cccccc}
N_{t}\left(a_{2} x\right) & = & a_{2} N_{t} x+a_{1} t x+2 a_{1} t^{2} x & N_{t}\left(\bar{a}_{2} x\right) & = & \bar{a}_{2} N_{t} x+\bar{a}_{1} t x+2 \bar{a}_{1} t^{2} x \\
(s-1)\left(a_{2} x\right) & = & a_{2}(s-1) x-\bar{a}_{1} s x & (s-1)\left(\bar{a}_{2} x\right) & = & \bar{a}_{2}(s-1) x+a_{1} s x \\
(t-1)\left(a_{2} x\right) & = & a_{2}(t-1) x+a_{1} t x & (t-1)\left(\bar{a}_{2} x\right) & = & \bar{a}_{2}(t-1) x+\bar{a}_{1} t x \\
N_{s}\left(a_{2} x\right) & = & a_{2} N_{s} x+\bar{a}_{1} s x+\bar{a}_{1} s^{2} x & N_{s}\left(\bar{a}_{2} x\right) & = & \bar{a}_{2} N_{s} x+a_{1} s x-a_{1} s^{2} x
\end{array}
$$

c) We have the following identity of functions on $V$ :

$$
\begin{aligned}
& N_{t} p_{2}=-a_{1} \quad N_{t} \bar{p}_{2}=-\bar{a}_{1} \\
& \begin{array}{rlcccc}
s p_{2}-p_{2}+q_{2}-t q_{2} & = & 0 & s \bar{p}_{2}-\bar{p}_{2}+\bar{q}_{2}-t \bar{q}_{2} & = & 0 \\
N_{s} q_{2} & = & \bar{a}_{1} & N_{s} \bar{q}_{2} & = & -a_{1}
\end{array} \\
& p_{3}+t p_{3}+t^{2} p_{3}-t p_{2}+t^{2} p_{2}=a_{1}-a_{2} \\
& \bar{p}_{3}+t \bar{p}_{3}+t^{2} \bar{p}_{3}-t \bar{p}_{2}+t^{2} \bar{p}_{2}=\bar{a}_{1}-\bar{a}_{2} \\
& q_{3}+s q_{3}+s^{2} q_{3}+s \bar{q}_{2}-s^{2} \bar{q}_{2}=a_{1}+\bar{a}_{1}+\bar{a}_{2} \\
& \bar{q}_{3}+s \bar{q}_{3}+s^{2} \bar{q}_{3}-s q_{2}+s^{2} q_{2}=\bar{a}_{1}-a_{1}-a_{2} \\
& p_{3}-s \bar{p}_{2}-q_{2}-\bar{q}_{2}+t \bar{q}_{2}-s p_{3}-q_{3}+t q_{3}=0 \\
& \bar{p}_{3}+s p_{2}+q_{2}-\bar{q}_{2}+t q_{2}-s \bar{p}_{3}-q_{3}+t q_{3}=0 .
\end{aligned}
$$


Proof: a) follows because $t$ and $s$ act trivially on $a_{1}$ and $\bar{a}_{1}$.

b) is easily verified, for example

$$
\begin{aligned}
N_{t}\left(a_{2} x\right)= & t^{2}\left(a_{2}\right) t^{2}(x)+t\left(a_{2}\right) t(x)+a_{2} x \\
& =\left(a_{2}+2 a_{1}\right) t^{2}(x)+\left(a_{2}+a_{1}\right) t(x)+a_{2} x=a_{2} N_{t} x+2 a_{1} t^{2} x+a_{1} t x .
\end{aligned}
$$

c) is explicitly verified on the $3^{6}$ elements of $V$ (the identities have been found by taking the vectors of values of these functions and finding linear relations between them).

Proposition B.4. The first term $\xi \cup V^{3}$ in the Charlap Vasquez description of the differential $d_{2}^{0,3}$ is given by

$$
\begin{aligned}
C & \mapsto\left(\alpha_{1} \alpha_{2}-\bar{\alpha}_{1} \bar{\alpha}_{2}, 0,-\alpha_{1} \bar{\alpha}_{2}-\bar{\alpha}_{1} \alpha_{2}\right) \\
D & \mapsto\left(\alpha_{1} \bar{\alpha}_{2}+\bar{\alpha}_{1} \alpha_{2}, 0, \alpha_{1} \alpha_{2}-\bar{\alpha}_{1} \bar{\alpha}_{2}\right)
\end{aligned}
$$

Proof: The three components are given by $N_{t} C_{1}^{1,2},-(s-1) C_{1}^{1,2}+(t-1) C_{2}^{1,2}$ and $N_{s} C_{2}^{1,2}$ and similarly for $D$. This calculation and the simplifications are done with the help of the last lemma. Finally we need that $a_{1} a_{2}$ and $-a_{2} a_{1}$ represent $\alpha_{1} \alpha_{2}$ and similarly for the other parts.

\section{REFERENCES}

[1] J.Aisbett, On $K_{3}\left(\mathbb{Z} / p^{n}\right)$ and $K_{4}\left(\mathbb{Z} / p^{n}\right)$, Memoirs AMS 329 (1985), 1-90

[2] M.Bökstedt, I.MAdSEn, Algebraic K-theory of local number fields: the unramified case, To appear.

[3] K.S.Brown, Cohomology of groups, GTM 87 Springer Verlag (1982)

[4] L.S.Charlap, A.T. Vasquez, The cohomology of group extensions, Trans. AMS 124 (1966), $24-40$

[5] R.K.Dennis, M.R.Stein, K 2 of discrete valuation rings, Adv.Math. 18 (1975), 182-238

[6] W.Dwyer, E.Friedlander, Algebraic and étale K-theory, Trans. AMS 292 (1985)

[7] L.Evens, E.M.Friedlander, On $\mathrm{K}_{*}\left(\mathbb{Z} / p^{2} \mathbb{Z}\right)$ and related homology groups, Trans. AMS 270 (1982), 1-46

[8] E.M.Friedlander, B.J.PARshall, On the cohomology of algebraic and related finite groups, Inv.Math. 74 (1983), 83-117

[9] R.Godement, Topologie algébrique et théorie des faisceaux, Hermann (1958)

[10] L.Hesselholt, I.Madsen, Topological cyclic homology of perfect fields and their dual numbers, To appear in Topology

[11] W. van Der Kallen, J.Stienstra, The relative $\mathrm{K}_{2}$ of truncated polynomial rings, J.Pure Appl.Math. 34 (1984), 277-289

[12] C.Kassel, Calcul algébrique de l'homologie de certains groupes de matrices, Journal of Algebra 90 (1983), 235-260

[13] M.Levine, The indecomposable $\mathrm{K}_{3}$ of fields, Ann.Scient.Ec.Norm.Sup. 22 (1989), 255-344

[14] E.Lluis-Puebla, On $\mathrm{K}_{3}\left(\mathbb{F}_{1}<[\approx] /\left(\approx^{\not \neq}\right)\right)$ and $\mathrm{K}_{3}(\mathbb{Z} / 9), p$ an odd prime, Memoirs AMS 329 (1985), 91-100

[15] I.A.PAnin, On a theorem of Hurewicz and K-theory of complete discrete valuation rings, Math. USSR Izvestija 29 (1987), 119-131

[16] D.Quillen, On the cohomology and K-theory of the general linear groups over a finite field, Annals Math. 96 (1972), 552-586

[17] P.Schneider, Über gewisse Galoiscohomologiegruppen, Math. Zeitschrift 168 (1979), 181-205

[18] J.P.SERRE, Local fields, GTM 67 Springer Verlag

[19] V.SnAith, On $K_{3}$ of dual numbers, Memoirs AMS 329 (1985), 101-200

[20] C.Soulé, Operations on étale K-theory. Applications, LNM 966 (1982), 271-303

[21] A.Susuin, On the K-theory of local fields, J.Pure Appl.Alg. 34 (1984), 301-318 
Harvard Department of Mathematics, 1 Oxford Street, Cambridge, MA 02138

E-mail address: geisser@math.harvard.edu 